\title{
WEAKLY NONUNIFORM THERMAL EFFECTS IN A POROUS CATALYST: ASYMPTOTIC MODELS AND LOCAL NONLINEAR STABILITY OF THE STEADY STATES*
}

\author{
FRANCISCO J. MANCEBO† AND JOSÉ M. VEGA $\dagger$
}

\begin{abstract}
This paper considers a first-order, irreversible exothermic reaction in a bounded porous catalyst, with smooth boundary, in one, two, and three space dimensions. It is assurned that the characteristic reaction time is sufficiently small for the chemical reaction to be confined to a thin layer near the boundary of the catalyst, and that the thermal diffusivity is large enough for the ternperature to be uniform in the reaction layer, but that it is not so large as to avoid significant thermal gradients inside the catalyst. For appropriate realistic limiting values of the several nondimensional parameters of the problenn, several time-dependent asymptotic models are derived that account for the chemical reaction at the boundary (that becomes essentially impervious to the reactant), heat conduction inside the catalyst, and exchange of heat and reactant with the surrounding unreacted fluid. These models possess asyrnmetrical steady states for symmetric shapes of the catalyst, and some of them exhibit a rich dynamic behavior that includes guasiperiodic phenomena. In one case, the linear stability of the steady states, and also the local bifurcation to quasi-periodic solutions via center manifold theory and normal form reduction, are analyzed.
\end{abstract}

Key words. porous catalysts, weakly nonlinear stability, normal forms

AMS(MOS) subject classifications. 35B32, 35K57, 80A30, $80 \mathrm{~A} 32$

1. Introduction and formulation. This paper deals with a well-known model for the evolution of the reactant concentration $u$ and of the temperature $v$ in a porous catalyst, in which a first-order, irreversible, exothermic reaction occurs. After suitable nondimensionalization (length is referred to a characteristic dimension of the catalyst, time is referred to the thermal diffusion time within the catalyst, and the reactant concentration and temperature are referred to their respective values at the external unreacted fluid), the principle of conservation applied to the reactant and to enthalpy leads to the following model [1, Vol. I]:

$$
\begin{aligned}
& L \partial u / \partial t=\Delta u-\phi^{2} u \exp (\gamma-\gamma / v) \quad \text { in } \Omega, \quad \partial u / \partial n=\sigma(1-u) \quad \text { at } \partial \Omega \text {, } \\
& \partial v / \partial t=\Delta v+\beta \phi^{2} u \exp (\gamma-\gamma / v) \quad \text { in } \Omega, \quad \partial v / \partial n=v(1-v) \text { at } \partial \Omega \text {, }
\end{aligned}
$$

for $t>0$, with appropriate initial conditions at $t=0$. Here $\Delta$ is the Laplacian operator, $n$ is the outward unit normal to the smooth boundary of the domain $\Omega \subset \mathbb{R}^{p}(p=1$, 2 , or 3), and all the parameters are positive. $\phi^{2}$ (Damköhler number) is the ratio of the reaction rate to the diffusion rate, $\gamma$ is the activation energy (or temperature) of the chemical reaction, $L$ (Lewis number) is the ratio of thermal to materiai diffusivity, $\beta$ (Prater number) is a measure of the chemical heat release ( $\beta L$ is a measure of the heat of reaction relative to the thermal energy of the catalyst), and $\sigma$ and $\nu$ (diffusional and thermal Biot numbers) are measures of the rates of mass and heat transfer between the surface of catalyst and the external fluid, relative to the rates of mass and heat transfer within the catalyst.

Usually, the thermal diffusivity of the solid (metallic or with metallic components) catalyst is very high, and, consequently, $\beta$ is quite small, $\nu$ is fairly small or of order 
unity (depending on the size of the catalyst), and $L$ may be targe. In addition, $\sigma / v$ is usually quite large because the exchange of heat (respectively, of mass) with the external fluid is much slower (respectively, faster) than through the structure (respectively, the pores) of the catalyst. The activation energy is of order unity or fairly large for most chemical reactions of industrial interest, and $\phi^{2}$ varies in a wide range (in particular, this parameter may be large in practice). Therefore the limit

$$
L \rightarrow \infty, \quad \gamma \beta \rightarrow 0, \quad \sigma / \nu \rightarrow \infty
$$

is realistic (although an additional assumption will be made below on the combined limit (1.3) to account for the most usual numerical values of the parameters).

If, in addition to (1.3), $\nu$ is assumed to be small, then the so-called isothermal models are obtained, in which the temperature inside the catalyst is uniform (but not necessarily equal to its value in the external fluid). A time-dependent isothermal model was first considered by Amundson and Raymond [2]; for a more recent analysis of these models, see [3], and [4], where they are formally derived (for a rigorous derivation of the isothermal models, see [30]), linear stability and Hopf bifurcation diagrams are obtained, and global stabikity properties are analyzed. For an analysis of the steady states of these models, with more general kinetic laws than the first-order Arrhenius law considered above, see [5], [31].

In this paper, we consider the limit (1.3) for $\nu \sim 1$. If $\phi^{2}=O(1)$, then the following completely isothermal model (the temperature $v$ is uniform inside the catalyst and equal to its value at the external fluid, $v=1$ ) is readily obtained in first approximation:

$$
\partial u / \partial \tau=\Delta u-\phi^{2} u \text { in } \Omega, \quad u=1 \text { at } \partial \Omega .
$$

Here the time variable is $\tau=t / L$. The linear problem (1.4) is not very interesting (a unique steady state exists that is globally, asymptotically stable). If the nonlinearity $\phi^{2} t \exp (\gamma-\gamma / v)$ in (1.1) and (1.2) is replaced by a more general one, of the type $\phi^{2} f(u, v)$, where $f:\left[0, \infty\left[^{2} \rightarrow \mathbb{R}\right.\right.$ is a positive smooth function (such that $\left.f(0, v) \equiv 0\right)$, then we must replace in $(1.4) \phi^{2} u$ by $\phi^{2} f(u, 1)$. The resulting model exhibits muttiple steady states if the function $u \rightarrow f(u, 1)$ is appropriate (see [6] for a review on these questions), but its dynamics is again fairly simple, since the solutions converge to the set of the steady states for large $\tau$; see [7].

The limit $v \sim 1, \phi^{2} \rightarrow \infty$ is more interesting. In the distinguished limit

$$
\phi^{2}-L \sim \beta^{-2}-\sigma^{2} \rightarrow \infty \text {, }
$$

the following basic model is derived in Appendix A:

$$
\begin{gathered}
\partial v / \partial t=\Delta v \text { in } \Omega, \\
\partial v / \partial n=\nu\left(1-v_{p}\right)+b \varphi^{2} \exp \left(\gamma-\gamma / v_{p}\right) \int_{-\infty}^{0} u_{p}(\zeta, \tau) d \xi \text { at each } p \in \partial \Omega,
\end{gathered}
$$

where, at each point $p \in \partial \Omega, v_{p}(t) \equiv v(p, t)$, and the function $u_{p}$ is given by

$$
\begin{gathered}
\lambda \partial u_{p} / \partial t=\partial^{2} u_{p} / \partial \zeta^{2}-\varphi^{2} u_{p} \exp \left(\gamma-\gamma / v_{p}\right) \quad \text { in }-\infty<\zeta<0, \\
u_{p}=0 \text { at } \zeta=-\infty, \quad \partial u_{p} / \partial \zeta=1-u_{p} \quad \text { at } \zeta=0 .
\end{gathered}
$$

Here the parameters $\varphi^{2}, b$, and $\lambda$, and the variable $\zeta$, are

$$
\varphi^{2}=\phi^{2} / \sigma^{2}, \quad b=\sigma \beta, \quad \lambda=L / \sigma^{2}, \quad \zeta=\sigma \eta,
$$

where $\eta$ is a coordinate along the outward unit normal to $\partial \Omega$.

The physical meaning of this model is the following. Since the characteristic reaction time is small compared with the diffusion time $\left(\phi^{2} \rightarrow \infty\right)$, the reactant is 
depleted in a thin reaction layer, of thickness $\phi^{-1}$, beside the boundary of $\Omega$. Also, the thermal diffusivity is sufficiently large $(\beta \rightarrow 0)$ for the temperature to be uniform, in the reaction layer, along each normal to $\partial \Omega$; thus the evolution of the reactant concentration in the reaction layer is given by (1.8) and (1.9), where $v$ is the local temperature (at each point of $\partial \Omega$ ) that does not depend on the inner space coordinate $\zeta$. The main difference with the isothermal limit is that here $(\nu \sim 1)$ the thermal diffusivity is not so large as to make the temperature uniform inside $\Omega$, where it now evolves according to the heat equation (1.6). In the nonlinear boundary condition (1.7), the total heat flux through each point of $\partial \Omega$ equals the heat exchange with the external fluid (the first term in the right-hand side) plus the total heat produced by the chemical reaction, in the reaction layer, along each normal to $2 \Omega$.

The model (1.6)-(1.9) may be further simplified because $\lambda$ is small most frequently in practice, since $\sigma / \nu \sim 100, L$ is not larger than 100, and we are assuming that $\nu \sim 1$. Although the ratio $\sigma / \nu$ may be much smaller in some cases (e.g., in hydrogen-rich systems, see [1]) we restrict ourselves to the limit $\lambda \rightarrow 0$, which will be considered in $\$ 2$, where the following distinguished limits will be analyzed:

$$
\begin{gathered}
\varphi^{2}-b \sim 1, \quad \lambda \rightarrow 0, \\
\varphi^{2} \sim 1 / b^{2} \sim \lambda \rightarrow 0 .
\end{gathered}
$$

In the limit (1.11), the model (1.6)-(1.9) will be reduced, in first approximation, to the heat equation (1.6) with the following nonlinear boundary condition:

$$
\partial v / \partial n=\nu(1-v)+b \varphi /[\varphi+\exp (\gamma / 2 v-\gamma / 2)] \text { at } \partial \Omega
$$

The problem (1.6) and (1.13) will henceforth be referred to as model 1. It was first considered by Pis'men and Kharkats [8], who obtained it from a physical problem (the so-called exterior problem of Pis'men and Kharkats; see [1]) that is somewhat similar to that considered here. The main feature of model 1 is that it exhibits up to four stable steady states (and five additional unstable ones) in one space dimension; it also possesses asymmetric steady states in symmetric domains. In $\$ 2.1$, we collect some resuits in the literature for related problems that apply to model 1 and derive two additional submodels that account for the limit $\gamma \rightarrow \infty$. One of these submodels applies in the limit

$$
\varphi^{2} \sim(\gamma b)^{2} \sim 1, \quad \lambda \rightarrow 0 .
$$

In the limit (1.12), the model (1.6)-(1.9) will be reduced (in $\$ 2.2)$ to the following model, that will henceforth be referred to as model 2 . That model is posed by the heat equation (1.6) with boundary conditions

$$
\partial v / \partial n=\nu\left(1-v_{p}\right)+B \Phi^{2} \exp \left(\gamma-\gamma / v_{p}\right) \int_{-\infty}^{0} u_{p}(\xi, t) d \xi \text { at each } p \in \partial \Omega
$$

where, at each point $p \in \partial \Omega, v_{p}(t) \equiv v(p, t)$, and the function $u_{p}$ is given by

$$
\begin{gathered}
\partial u_{p} / \partial t=\partial^{2} u_{p} / \partial \xi^{2}-\Phi^{2} u_{p} \exp \left(\gamma-\gamma / v_{p}\right) \text { in }-\infty<\xi<0, \\
u_{p}=0 \text { at } \xi=-\infty, \quad u_{p}=1 \quad \text { at } \xi=0 .
\end{gathered}
$$

Here the parameters $B$ and $\varphi^{2}$, and the variable $\xi$, are

$$
B=b \sqrt{\lambda}=\beta \sqrt{L}, \quad \Phi^{2}=\varphi^{2} / \gamma=\phi^{2} / L, \quad \xi=\zeta \sqrt{\lambda}=\eta \sqrt{L}
$$

where, again, $\eta$ is a coordinate along the outward unit normal to $2 \Omega$. Observe that model 2 differs from the basic model only in the boundary conditions $(1.16)$ at $\xi=0$ 
and in the fact that it depends on three nondimensional parameters the basic model depends on four parameters). In $\$ 2.2$ we give two additional submodels of model 2 , accounting for the limit $\gamma \rightarrow \infty$. One of these submodels applies in the limit $\Phi \sim 1$, $B \sim \gamma^{1} \rightarrow 0$, or $($ see $(1.12),(1.17))$

$$
\varphi^{2} \sim 1 /(\gamma b)^{2} \sim \lambda \rightarrow 0
$$

Note that the limits (1.12) and (1.12) are realistic. If, for example, $\nu=1, \phi=10$, $o=L=100$, and either $(\gamma, \beta)=(5, .05)$ or $(\gamma, \beta)=(20, .005)$, then either $\varphi^{2}=\lambda=.01$, $b^{-2}=.04$ or $\varphi^{2}=\lambda=(\gamma \beta)^{-2}=.01$, and one of the limits $(1.12)$ or $\left(1.12^{\prime}\right)$ should be expected to apply. Observe that these two sets of values of the parameters in (1.1) and (1.2) are realistic (see, e.g., [1, Vol. I, pp.94-97]).

Model 2 exhibits the same features as model 1 in connection with its steady states, which will be analyzed in $\$ 3.1$, for the one space dimension case. However, the dynamic behavior of model 2 is more interesting than that of model 1 . In $\$ 3.2$ an analysis of the linear stability of the steady states in one space dimension will be given. In particular, it will be seen that model 2 exhibits the following degeneracies $\left(\omega_{1}, \omega_{2}, \cdots\right.$ are the eigenvalues of the linearized problem around a steady state with zero real part): (i) $\omega_{1}=0, \omega_{2,3}= \pm i \Omega, \Omega \neq 0$, (ii) $\omega_{1,2}= \pm i \Omega_{1}, \omega_{3,4}= \pm i \Omega_{2}, \Omega \neq 0, \Omega_{2} \neq 0$, (iii) $\omega_{1}=0$ (triple), (iv) $\omega_{1}=0$ (double), $\omega_{2,3}= \pm i \Omega, \Omega \neq 0$, and (v) $\omega_{1}=0, \omega_{2,3}= \pm i \Omega_{1}, \omega_{4,5}= \pm i \Omega_{2}$, $\Omega_{1} \neq 0, \Omega_{2} \neq 0$. These degeneracies are known to lead to quasi-periodic phenomena, which is a well-known route to chaos [9], [10]. The normal forms of the (codimensiontwo) degeneracies (i) and (ii) are completely analyzed in [9]. For an analysis of the normal forms of the (codimension-three) degeneracies (iii)-(v), see [11]-[13]. See also [14] for an asymptotic analysis of the nondegeneracy (iii) in a particular case, allowing a description of chaotic solutions by analytical means.

In $\$ 4$ we obtain the coefficients of the normal form of the degeneracy (i), above, for model 2 via center manifold theory. That analysis will show that model 2 possesses quasi-periodic solutions that bifurcate from a family of periodic ones. That result makes it reasonable to expect chaotic behavior. There is a second reason for that expectation. The standard model of continuous stirred tank reactor consists of a pair of ordinary differential equations (ODEs) and exhibits nothing more interesting than periodic solutions. However, if an external thermal capacitance is added, the resuiting model possesses chaotic solutions (that, by the way, bifurcate from a family of periodic ones via the degeneracy (i), above); see [15]. In the same way, model 2 may be seen as a result of adding to an isothermal model the heat conduction effect in the porous body, which acts as a thermal capacitance for the exothermic chemical reaction (that occurs only in the reaction layer). Thus, from a physical point of view, model 2 is somewhat similar to that considered in [15]. Nevertheless, the expectable chaotic behavior of model 2 will not be pursued any further in this paper.

2. Models 1 and 2. Here we consider the limit $\lambda \rightarrow 0$ for the basic model (1.6)-(1.9). In fact, in the distinguished limits (1.11) and (1.12), models 1 and 2, posed by (1.6), (1.13) and by (1.6), (1.14)-(1.16), respectively, are obtained. In addition, we consider some properties of models 1 and 2 .

2.1 The Pis'men-Kharkats model, or model 1. In the limit (1.11), if we let $\lambda \rightarrow 0$ formally in (1.6)-(1.9), then we obtain in first approximation the model posed by (1.6) and (1.7), where, at each point of $\partial \Omega, u$ is given by

$$
\begin{gathered}
0=\partial^{2} u / \partial \zeta^{2}-\varphi^{2} u \exp (\gamma-\gamma / v) \quad \text { in }-\infty<\zeta<0, \\
u=0 \quad \text { at } \zeta=-\infty, \quad \partial u / \partial \zeta=1-u \text { at } \zeta=0 .
\end{gathered}
$$


The linear problem $(2.1),(2.2)$ is readily integrated to obtain $u$ ( $v$ is a parameter in (2.1)). A further substitution into (1.7) yields (1.13), and model 1, posed by (1.6), (1.13), is obtained.

Observe that if, instead of the basic model, we consider the more general one described in the last paragraph of Appendix A, then, in the limit (1.11), we obtain the model posed by (1.6), with boundary conditions

$$
\partial v / \partial n=v(1-v)+b \varphi h(\varphi, v) \text { at } \partial \Omega \text {, }
$$

where

$$
h(\varphi, v) \equiv\left[2 \int_{0}^{u_{0}(\varphi, v)} f(z, v) d z\right]^{1 / 2},
$$

and, for each $v \geq 0$ and each $\varphi>0, u_{0}(\varphi, v)$ is the unique solution of the equation

$$
1-u_{0}=\varphi\left[2 \int_{0}^{u_{0}} f(z, v) d z\right]^{1 / 2} .
$$

The most relevant properties (steady states and their stability, dynamics) of model 1 (posed by (1.6), (1.13)) in one space dimension $(\Omega=]-1,1[\subset \mathbb{R}$ ) either are well known or may be established by applying results in the literature for related problems. In particular, model 1 possesses generically one or three symmetric (and uniform) steady states and, for appropriate values of the parameters, two or six asymmetric steady states, as shown by Pis'men and Kharkats [14] (see also [16]). The stability of the steady states was also analyzed in [14], and it was found that the stable ones are exactly the following: (i) the minimal and maximal untform steady states, and (ii) two of the asymmetric steady states when there are six. The domains of attraction of the stable steady states (for a slightly different model suggested by a related problem) were analyzed by Aronson and Peletier [17] and by Aronson [18]. In addition, Ball and Peletier [19] (see also [20]) proved (for a somewhat more general model) that, for arbitrary initial conditions, the soiution stabilizes (i.e., approaches a steady state) as $t \rightarrow \infty$. The generalization of these properties to two and three space dimensions is presented elsewhere [21].

Finally, we consider the limit $\gamma \rightarrow \infty$ for model 1 . Two distinguished limits must be considered. If

$$
\varphi \sim \exp (-\gamma / 2), \quad v \sim 1, \quad v \sim b \sim \gamma \rightarrow \infty,
$$

then, in first approximation, we obtain the model posed by (1.6), with boundary conditions

$$
\partial v / \partial n=-v v+b_{1} \varphi_{1} /\left[\varphi_{1}+\exp (1 / 2 v)\right] \text { at } \partial \Omega .
$$

To obtain that model, replace $v$ by $\gamma w, b$ by $\gamma b_{1}$, and $\varphi$ by $\varphi_{1} \exp (-\gamma / 2)$ in $(1.6)$, (1.13), divide by $\gamma$, and let $\gamma \rightarrow \infty$. That model is not quite significant, since it involves very large temperatures in the catalyst, and this must be avoided in practice for technical reasons.

The second distinguished limit is

$$
\nu \sim \varphi \sim 1, \quad b \sim|v-1| \sim y^{-1} \rightarrow 0 .
$$

Now, by replacing $v$ by $1+v / \gamma$ and $b$ by $b_{2} / \gamma$ in (1.6), (1.13), multiplying by $\gamma$, and letting $\gamma \rightarrow \infty$, we obtain, in first approximation, the model posed by (1.6) with boundary conditions

$$
\partial v / \partial n=-\nu v+b_{2} \varphi /[\varphi+\exp (-v / 2)] \text { at } \partial \Omega
$$


2.2. Model 2. This model is posed by (1.6), (1.14)-(1.16), and it is obtained from the basic model (1.6)-(1.9) in the distinguished limit (1.12), by introducing the variables and parameters (1.17) into (1.6)-(1.9) and letting $\lambda \rightarrow 0$.

As $\gamma \rightarrow \infty$, we obtain two distinguished submodels of model 2. The first one, which (as above) is not quite significant, is obtained in the limit $\Phi \sim \exp (-\gamma / 2), v \sim B \sim \gamma \rightarrow \infty$ by replacing $v$ by $\gamma v, B$ by $\gamma B_{1}$, and $\Phi^{2} \exp (-\gamma)$ in (1.6), (1.14)-(1.16), dividing (1.6) and $(1.14)$ by $\gamma$, and letting $\gamma \rightarrow \infty$. The second submodel is obtained in the limit $\nu \sim \Phi \sim 1, B \sim|v-1| \sim \gamma^{-1} \rightarrow 0$, by replacing $v$ by $1+v / \gamma$ and $B$ by $B_{2} / \gamma$ in $(1.6)$, (1.14)-\{1.16), multiplying (1.6) and (1.14) by $\gamma$, and letting $\gamma \rightarrow \infty$.

3. The steady states of model 2 and their linear stability in one dimension. Here we consider model 2 in one space dimension. It is posed by

$$
\begin{gathered}
\partial v / \partial t=\partial^{2} v / \partial x^{2} \text { in }-1<x<1 \\
\partial v / \partial x= \pm\left[\nu\left(1-v_{ \pm}\right)+B \Phi^{2} \exp \left(\gamma-\gamma / v_{ \pm}\right) \int_{-\infty}^{0} u_{ \pm}(\xi, t) d \xi\right] \text { at } x= \pm 1
\end{gathered}
$$

where $v_{ \pm}(t) \equiv v( \pm 1, t)$ and the functions $u_{+}$and $u_{-}$are given by

$$
\begin{gathered}
\partial u_{ \pm} / \partial t=\partial^{2} u_{ \pm} / \partial \xi^{2}-\Phi^{2} u_{ \pm} \exp \left(\gamma-\gamma / v_{ \pm}\right) \quad \text { in }-\infty<\xi<0, \\
u_{ \pm}=0 \quad \text { at } \xi=-\infty, \quad u_{ \pm}=1 \quad \text { at } \xi=0 .
\end{gathered}
$$

3.1. The steady state solutions. The steady states of $(3.1)-(3.4)$ are readily seen to be given by

$$
v=\left(v_{1}-v_{-}\right) x / 2+\left(v_{+}+v_{-}\right) / 2
$$

where $v_{ \pm} \equiv v( \pm 1)$ satisfy

$$
\begin{aligned}
& v_{+}=(1+2 \nu) v_{-}-2 \nu-2 B \Phi \exp \left(\gamma / 2-\gamma / 2 v_{-}\right), \\
& v_{-}=(1+2 \nu) v_{+}-2 \nu-2 B \Phi \exp \left(\gamma / 2-\gamma / 2 v_{+}\right)
\end{aligned}
$$

By applying standard bifurcation techniques (see, e.g., [22]) to (3.5), (3.6), it is seen that, for fixed values of $\nu$ and $\gamma$, the response curve $v_{+}-\Phi$ is as one of the plots of Fig. 1. The $S$-shaped part of the response curve corresponds to steady states such that ( $v_{-}=v_{+}$and) the temperature is uniform, while the remaining part of the response curve corresponds to nonuniform temperature profiles $\left(v_{-} \neq v_{+}\right)$. Observe that, for appropriate values of $\nu$ and $\gamma$, the response curve can exhibit up to six bending points (i.e., the points $1,2,5,5^{\prime}, 6$, and $6^{\prime}$ ) and two pitchfork bifurcation points (i.e., the points 3 and 4). In Fig. 2, the common boundary of regions $I$ and $I I$ (where the bending points 1 and 2 coalesce) is given by $\gamma=8$, as obtained by eliminating $v$ and $B \Phi$ from the following system of equations:

$$
F(\nu, \gamma, B \Phi, v)=0, \quad F_{v}(\nu, \gamma, B \Phi, v)=0, \quad F_{v v}(\nu, \gamma, B \Phi, v)=0,
$$

where the function $F$ is defined by

$$
F(\nu, \gamma, \varphi, v) \equiv \nu(1-v)+\varphi \exp (\gamma / 2-\gamma / 2 v) .
$$

The common boundary of regions II and III (where the pitchfork bifurcation points 3 and 4 coalesce) is given by $\gamma=8(1+1 / v)$, as obtained by eliminating $v$ and $B \Phi$ from the following system:

$$
\begin{gathered}
F(\nu, \gamma, B \Phi, v)=0, \quad F_{v}(\nu, \gamma, B \Phi, v)=0 \\
F_{\varphi v}(\nu, \gamma, B \Phi, v)=F_{\varphi}(\nu, \gamma, B \Phi, v) F_{v v}(\nu, \gamma, B \Phi, v),
\end{gathered}
$$



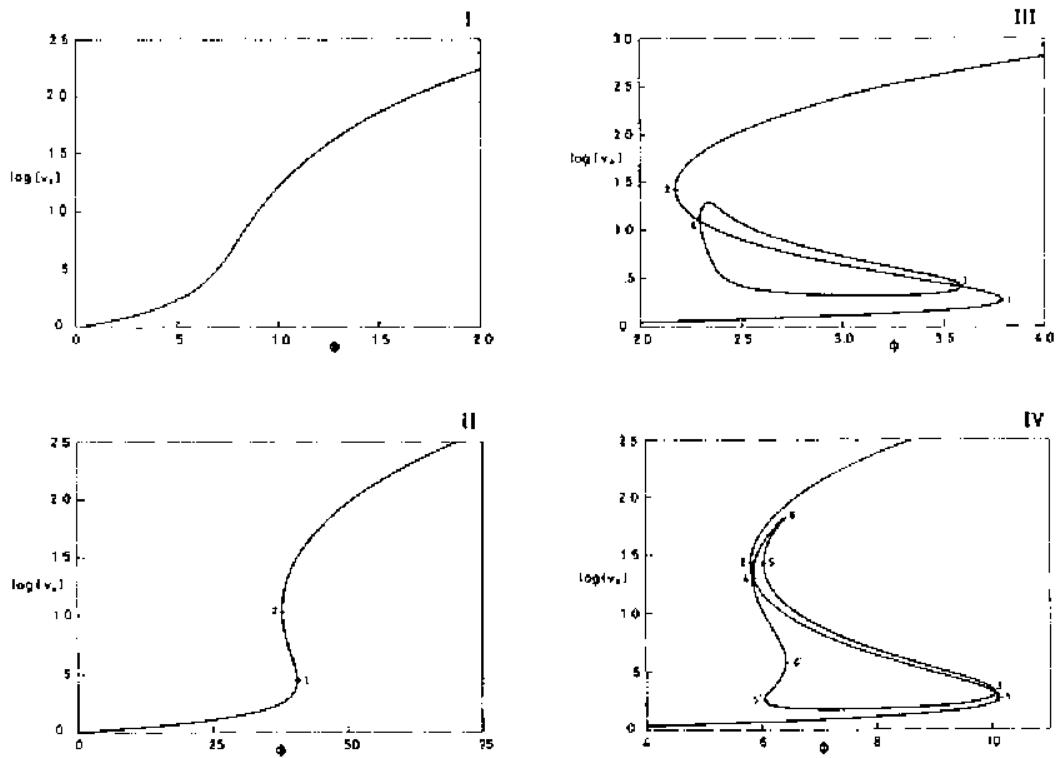

F[G. 1. Steady state response curves of model 1 for representative points of the regions I, II, III, and IV in Fig. 2. (I: $(\nu, \gamma)=(3.5,6) ; \mathrm{II}:(\nu, \gamma)=(3.5,8.75) ; 1 \mathrm{II}:(\nu, \gamma)=(4.48, \cdots, 11) ; \mathrm{IV}:(\nu, \gamma\}=(12,11)$.)

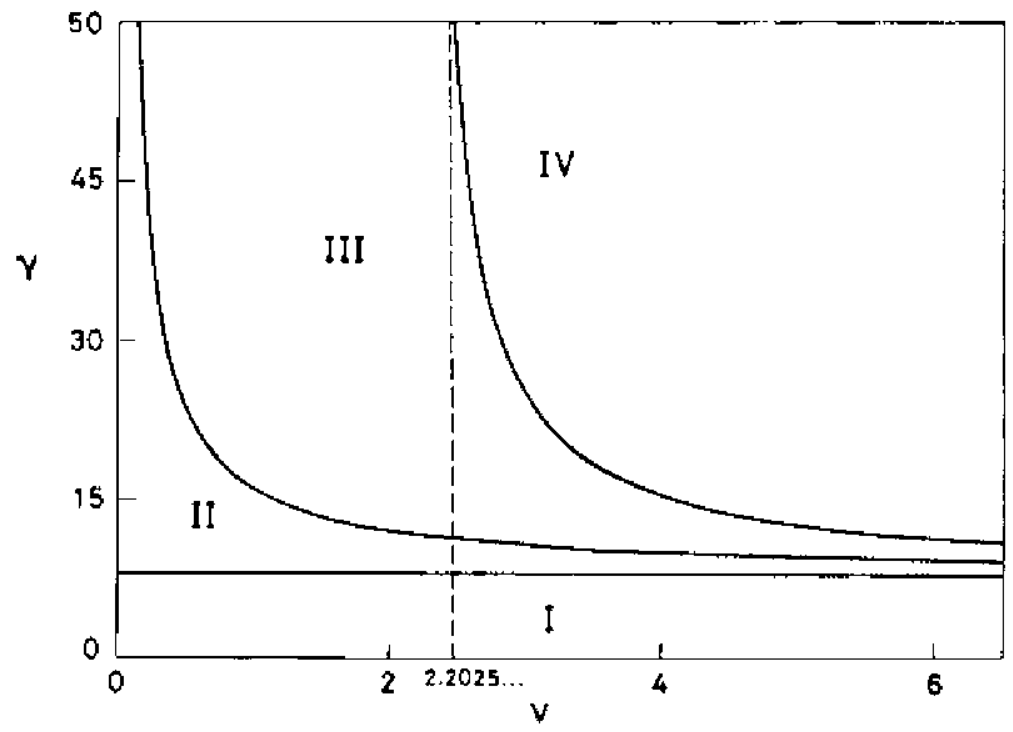

FIC. 2. Boundary curves in the plane $\gamma-\nu$, of the regions I, II, III, and IV.

where the function $F$ is as defined in (3.7). Finally, the common boundary of regions III and IV (where the two pairs of bending points 5-6 and $5^{\prime}-6^{\prime}$ coalesce simuitaneously) is obtained (numerically) by eliminating $v_{-}, v_{+}$, and $B \Phi$ from the following system of equations:

$$
\begin{gathered}
v_{+}-v_{-}+2 F\left(\nu, \gamma, B \Phi, v_{-}\right)=0, \quad v_{-}-v_{+}+2 F\left(\nu, \gamma, B \Phi, v_{+}\right)=0, \\
F_{v}\left(\nu, \gamma, B \Phi, v_{-}\right)+F_{v}\left(\nu, \gamma, B \Phi, v_{+}\right)=2 F_{v}\left(\nu, \gamma, B \Phi, v_{-}\right) F_{v}\left(\nu, \gamma, B \Phi, v_{+}\right), \\
F_{v v}\left(\nu, \gamma, B \Phi, v_{+}\right) / F_{v v}\left(\nu, \gamma, B \Phi, v_{-}\right)=\left[F_{v}\left(\nu, \gamma, B \Phi, v_{+}\right) / F_{v}\left(\nu, \gamma, B \Phi, v_{-}\right)\right]^{3},
\end{gathered}
$$

where the function $F$ is defined as in (3.7). 
3.2. Linear stability of the uniform steady states. Now we study the linear stability of the steady states of (3.1)-(3.4) with uniform temperature profiles, which henceforth will be called uniform steady states. The linear stability of the remaining steady states is analyzed in a similar way.

The (fairly involved) complete parametric results of the linear stability analysis is presented in the Ph.D. thesis of the first author [23]. Here, for brevity, we focus on locating degeneracies (of the linearized eigenvalue problem) of codimenston greater than one, where local bifurcation into (two- and three-dimensional) tori is expected to occur [9]-[13]. One such bifurcation is considered in $\$ 4$.

For the uniform steady states, the temperature $v_{s}$ is independent of $x$, and the functions $u_{s+}$ and $u_{s-}$ (giving the concentration profiles at the reaction layers) at $x=-1$ and at $x=1$ are identical. $v_{s}$ and $u_{s} \equiv u_{s \pm}$ are given by

$$
v_{s}=\left(\nu+B \Phi_{s}\right) / \nu, \quad u_{s}=\exp \left(\Phi_{s} \xi\right),
$$

in terms of the parameter

$$
\Phi_{s}=\Phi \exp \left[\gamma\left(v_{s}-1\right) / 2 v_{s}\right]
$$

The linearized problem around the steady state has nontrivial solutions $v-v_{s}=$ $V(x) \exp (\omega t), u_{ \pm}-u_{s}=U_{ \pm}(x) \exp (\omega t)$ at $x= \pm 1$ if and oniy if $\omega$ satisfies one of the following equations:

$$
\begin{aligned}
& \nu+\sqrt{\omega} \tanh \sqrt{\omega}-\left(\gamma B \Phi_{s} / v_{s}^{2}\right)\left[1-\Phi_{s}^{2} / \omega+\Phi_{s}^{3} /\left(\omega \sqrt{\Phi_{s}^{2}+\omega}\right)\right]=0 \\
& \nu+\sqrt{\omega} \operatorname{coth} \sqrt{\omega}-\left(\gamma B \Phi_{s} / v_{s}^{2}\right)\left[1-\Phi_{s}^{2} / \omega+\Phi_{s}^{3} /\left(\omega \sqrt{\Phi_{s}^{2}+\omega}\right)\right]=0 .
\end{aligned}
$$

When (3.10) (respectively, (3.11)) holds, then the function $x \rightarrow V(x)$ is even (respectively, odd), and the perturbed temperature profiles are symmetric (respectively, antisymmetric) with respect to $x=0$.

Let us now consider the marginally stable steady states, i.e., these steady states such that either (3.10) or (3.11) are satisfied with $\omega= \pm i \Omega$, with $\Omega$ real and nonnegative. We first analyze (3.10), which has the solution $\omega=0$ if and only if

$$
\gamma=2\left(\nu+B \Phi_{s}\right)^{2} / \nu B \Phi_{s} .
$$

That equation provides the bending points 1 and 2 of the response curves of Fig. 1 . Also, (3.10) has the solutions $\omega= \pm i \Omega$, with $\Omega>0$ if and only if the following equations hold:

$$
\gamma=\frac{2\left(\nu+B \Phi_{s}\right)^{2} \Omega \sqrt{\Omega^{2}+\Phi_{s}^{4}}}{\nu^{2} B \Phi_{s}\left(2 \Omega \sqrt{\Omega^{2}+\Phi_{s}^{4}}-\Phi_{s}^{3} b\right)}\left\{\sqrt{\frac{\Omega}{2}} \frac{\sinh \sqrt{2} \bar{\Omega}-\sin \sqrt{2 \Omega}}{\cosh \sqrt{2 \Omega}+\cos \sqrt{2 \Omega}}+\nu\right],
$$

$$
\frac{2 \Omega \sqrt{\Omega^{2}+\Phi_{s}^{4}}-\Phi_{s}^{3} b}{\Phi_{s}^{2}\left[2 \sqrt{\Omega^{2}}+\Phi_{s}^{4}-\Phi_{s} a\right]}=\frac{\sinh \sqrt{2 \Omega}-\sin \sqrt{2 \Omega}}{\sinh \sqrt{2 \Omega}+\sin \sqrt{2 \Omega}}+\nu \sqrt{\frac{2}{\Omega}} \frac{\cosh \sqrt{2 \Omega}+\cos \sqrt{2 \Omega}}{\sinh \sqrt{2 \Omega}+\sin \sqrt{2 \Omega}}
$$

where

$$
a \equiv \sqrt{2}\left(\Phi_{s}^{2}+\sqrt{\Omega^{2}+\Phi_{s}^{4}}\right)^{1 / 2}, \quad b \equiv \sqrt{2}\left(\sqrt{\Omega^{2}+\Phi_{s}^{4}}-\Phi_{s}^{2}\right)^{1 / 2} .
$$

Those equations provide Hopf bifurcation points of the $S_{*}$ shaped part of the response curves of Fig. 1.

For fixed values of $\nu>0$ and $B>0,(3.12)$ defines (respectively, (3.13), (3.14) define) a curve in the plane $\gamma-\Phi$, that is as the curve $C_{1}$ (respectively, curve $C_{2}$ ) in 
one of the sketches of Fig. 3. (For appropriate values of $\nu$, there are isolas that bifurcate from the curve $C_{2}$ (see [23]). These isolas are not shown in the sketches of Fig. 3 because they piay no role in the analysis below.) Both curves always intersect at a point $p_{1}$, which corresponds to the limit $\Omega \rightarrow 0$ in (3.13), (3.14). The coordinates of $p_{1}$ are

$$
\Phi_{s 1}=\sqrt{3 \nu / 4}, \quad \gamma_{1}=2\left(\nu+B \Phi_{s 1}\right)^{2} / \nu B \Phi_{s 1} .
$$

Curves $C_{1}$ and $C_{2}$ are tangent at $p_{1}$ if and only if $\nu=10 / 3$. If $\nu \neq 10 / 3$ and $B>0$, then at $p_{1}$ the linearized problem around the steady state possesses a double eigenvalue $\omega=0$, which is geometrically simple. This is the so-called saddle-node degeneracy, which has codimension two. For a complete analysis of its universal unfolding, see, e.g., [9]. If $\nu=10 / 3$ and $B>0$, then at $p_{1}$ the linearized problem around the steady state possesses a triple eigenvalue $\omega=0$, which is geometrically simple. This degeneracy is of codimension three and is partially analyzed in [11], where it is shown that it leads to bifurcation to two- and three-dimensional tori. Observe that these two degeneracies, which occur at point $p_{1}$ of Fig. 3, correspond to the following situation: a Hopf bifurcation point of the $S$-shaped part of the response curve of Fig. 1 approaches one of the bending points 1 or 2 and disappears.

A second point of intersection of curves $C_{1}$ and $C_{2}$ (point $p_{2}$ in Fig. 3) exists if and only if $\nu>10 / 3$ and $B>0$. The coordinates of $p_{2}$ are $\Phi_{s 2}$ and $\gamma_{2}=$ $2\left(\nu+B \Phi_{s 2}\right)^{2} / \nu B \Phi_{s 2}$, where, for each $\nu>10 / 3, \Phi_{s 2}>0$ is the unique solution of the equation that results when $\Omega$ is eliminated from the following system of equations:

$$
\begin{gathered}
\frac{\Omega \sqrt{\Omega^{2}+\Phi_{s}^{4}}-\Phi_{s}^{3} b}{\Phi_{s}^{2}\left(2 \sqrt{\Omega^{2}+\Phi_{s}^{4}}-\Phi_{s} a\right)}=\frac{\sinh \sqrt{2 \Omega}-\sin \sqrt{2 \bar{\Omega}}}{\sinh \sqrt{2 \Omega}+\sin \sqrt{2 \bar{\Omega}}}, \\
\nu=\frac{\Omega \sqrt{\Omega^{2}+\Phi_{s}^{4}}}{\Phi_{s}^{2}\left(2 \sqrt{\Omega^{2}+\Phi_{s}^{4}}-\Phi_{s} a\right)} \sqrt{\frac{\Omega}{2} \frac{\sinh \sqrt{2 \Omega}+\sin \sqrt{2 \Omega}}{\cosh \sqrt{2 \bar{\Omega}}+\cos \sqrt{2 \Omega}},}
\end{gathered}
$$
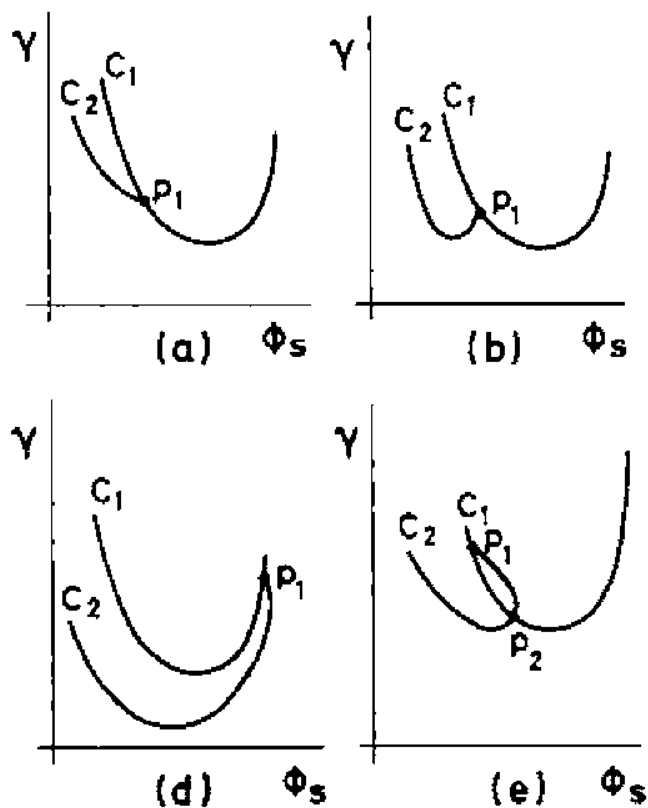
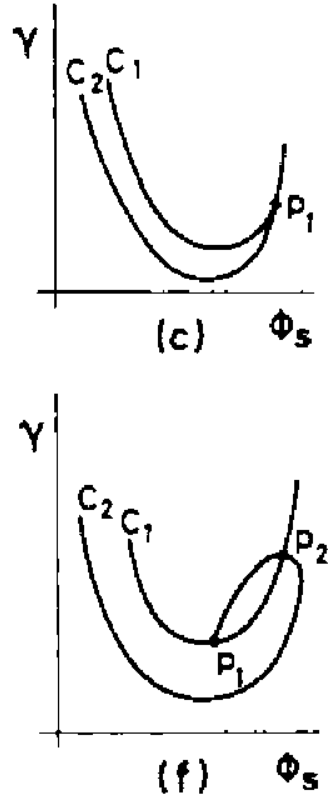
$B$ and $\nu$.

Frc. 3. Curves of neutral stability points of model 2 (under symmetric perturbations) for fixed values of 
where $a$ and $b$ are as defined in (3.15). At $p_{2}$ the linearized problem around the steady state has a simple eigenvalue $\omega=0$ and a pair of complex (simple) eigenvalues $\omega= \pm i \Omega$, with $\Omega \neq 0$. This is a codimension-two degeneracy whose universal unfolding has been completely analyzed [9]. In $\$ 4$ we study the behavior of (3.1)-(3.4) near that degeneracy. Observe that the degeneracy corresponds to the following situation: a Hopf bifurcation point of the response curve of Fig. 1 crosses one of the bending points 1 or 2 .

Let us now consider the eigenvalues of the linearized problem around the steady state that are solutions of (3.11). Equation (3.11) has the solution $\omega=0$ if and only if

$$
\gamma=2\left(\nu+1+B \Phi_{s}\right)^{2} /(v+1) B \Phi_{s} .
$$

That equation provides the pitchfork bifurcation points 3 and 4 of the sketches of Fig. 1. Also, (3.11) has the solutions $\omega= \pm i \Omega$, with $\Omega>0$, if and only if

$$
\begin{gathered}
\gamma=\frac{2\left(\nu+B \Phi_{s}\right)^{2} \Omega \sqrt{\Omega^{2}+\Phi_{s}^{4}}}{\nu^{2} B \Phi_{s}\left(2 \Omega \sqrt{\Omega^{2}+\Phi_{s}^{4}}-\Phi_{s}^{3} b\right)}\left[\sqrt{\left.\frac{\Omega}{2} \frac{\sinh \sqrt{2 \Omega}+\sin \sqrt{2 \Omega}}{\cosh \sqrt{2 \Omega}-\cos \sqrt{2 \Omega}}+\nu\right],}\right. \\
\frac{2 \Omega \sqrt{\Omega^{2}+\Phi_{s}^{4}}-\Phi_{s}^{3} b}{\Phi_{s}^{2}\left(2 \sqrt{\Omega^{2}+\Phi_{s}^{4}}-\Phi_{s} a\right)}=\frac{\sinh \sqrt{2 \Omega}+\sin \sqrt{2 \Omega}}{\sinh \sqrt{2 \Omega}-\sin \sqrt{2 \Omega}}+\nu \sqrt{\frac{2}{\Omega}} \frac{\cosh \sqrt{2 \Omega}-\cos \sqrt{2 \Omega}}{\sinh \sqrt{2 \Omega}-\sin \sqrt{2 \Omega}},
\end{gathered}
$$

where $a$ and $b$ are defined in (3.15).

For fixed values of $\nu>0$ and $B>0,(3.19)-(3.21)$ define two curves of the plane $\Phi_{s}-\gamma, C_{1}^{\prime}$ and $C_{2}^{\prime}$, which are qualitatively similar to the curves $C_{1}$ and $C_{2}$ of the sketches of Fig. 2. Here we are interested only in analyzing the intersections of the curve $C_{2}^{\prime}$ with the curves $C_{1}$ and $C_{2}$ (i.e., the points $p_{3}$ and $p_{4}$ of the sketches of Fig. 4). To this end, first observe that the curve $C_{1}$ is always below the curve $C_{1}^{\prime}$, as obtained by comparing (3.12) and (3.19). Also, as $\Phi_{s} \rightarrow 0$, the asymptotic behavior of the curves $C_{1}, C_{2}$, and $C_{2}^{t}$ is given by

$$
\gamma_{C_{1}}=2 \nu / B \Phi_{s}+O(1), \quad \gamma_{c_{2}}=\nu / B \Phi_{s}+O(1), \quad \gamma_{C_{2}^{*}}=(\nu+1) / B \Phi_{s}+O(1) .
$$

Then, for sufficiently small $\Phi_{s}$, the curve $C_{2}$ is below the curves $C_{2}^{\prime}$ and $C_{1}$ if $\nu>0$ and $B>0$, while the curve $C_{1}$ is below (respectively, above) the curve $C_{2}^{\prime}$ if $0<\nu<1$ (respectively, if $\nu>1$ ). Finally, the curves $C_{1}$ and $C_{2}^{\prime}$ intersect at a point $p_{3}$ (see

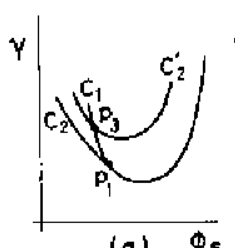

(a)

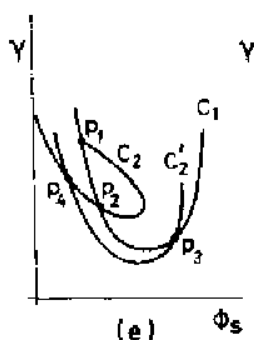

(e)

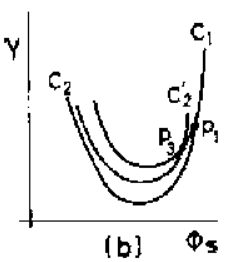

(b)

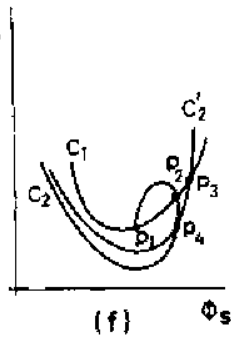

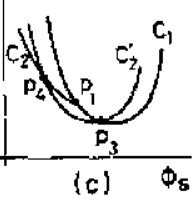
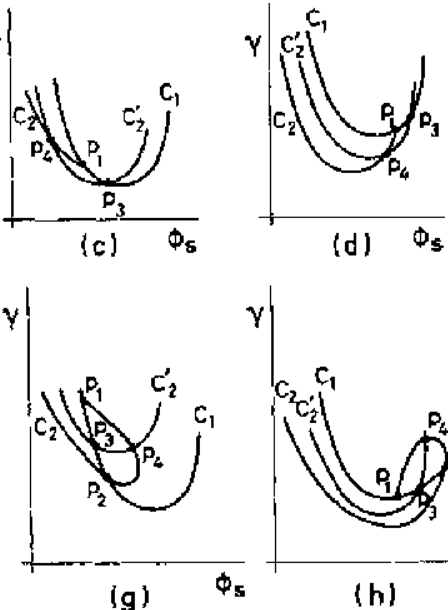

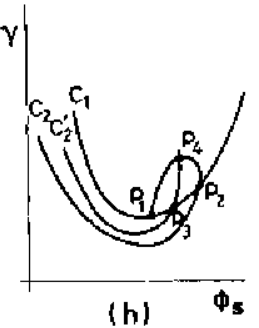

(b)

FIG. 4. Curves of reutral stability points of model 2 when asymmetric periurbations are considered. 
Fig. 4) if and only if $v>1$ and $B>0$. The coordinates of $p_{3}$ are $\Phi_{s 3}$ and $\gamma_{3}=$ $2\left(y+B \Phi_{s 3}\right)^{2} / \nu B \Phi_{s 3}$, where $\Phi_{s 3}$ is the unique solution of the equation that results when $\Omega$ is eliminated in the following system of equations:

$$
\begin{gathered}
\frac{\Omega \sqrt{\Omega^{2}+\Phi_{s}^{4}}-\Phi_{s}^{3} b}{\Phi_{s}^{2}\left(2 \sqrt{\Omega^{2}+\Phi_{s}^{4}}-\Phi_{s} a\right)}=\frac{\sinh \sqrt{2 \Omega}+\sin \sqrt{2 \Omega}}{\sinh \sqrt{2 \Omega}-\sin \sqrt{2 \Omega}}, \\
\nu=\frac{\Omega \sqrt{\Omega^{2}+\Phi_{s}^{4}}}{\Omega \sqrt{\Omega^{2}+\Phi_{s}^{4}}-\Phi_{s}^{3} b} \sqrt{\frac{\Omega}{2}} \frac{\sinh \sqrt{2 \Omega}+\sin \sqrt{2 \Omega}}{\cosh \sqrt{2 \bar{\Omega}}-\cos \sqrt{2 \Omega}},
\end{gathered}
$$

where $a$ and $b$ are as defined in (3.15). A plot of the first coordinate of the points 1 , 2 , and 3 (the last two of them as defined above) is given Fig. 5. If $0<v \leqq 1$ and $B>0$, then points 2 and 3 do not exist. If $1<v<\nu_{0}=1.8466 \cdots$, then $\Phi_{s 3}<\Phi_{s 1}$, while, if $\nu>\nu_{0}$, then $\Phi_{s 3}>\Phi_{s 1}$. The plots of $\Phi_{s 2}$ and $\Phi_{s 3}$ (the latter exists only if $\nu>10 / 3$ ) intersect infinitely often, for a sequence of values of $\nu, \nu_{1}=5.1482 \cdots<\nu_{2}=$ $8.5523 \cdots<\nu_{3}<\nu_{4}<\cdots$, as seen by an asymptotic analysis of the systems $(3.17)-(3.18)$ and $(3.22),(3.23)$; the asymptotic behavior of $y_{k}$ is given by

$$
v_{k}=(k \pi+.4695 \cdots) \times 1.0857 \cdots+o(1) \text { as } k \rightarrow \infty \text {. }
$$

From these properties the following conclusions hold:

(i) If $B>0$ and $0<\nu \leqq 1$, then the curve $C_{2}^{\prime}$ intersect neither the curve $C_{1}$ nor the curve $C_{2}$ (that is below the curve $C_{1}$ ).

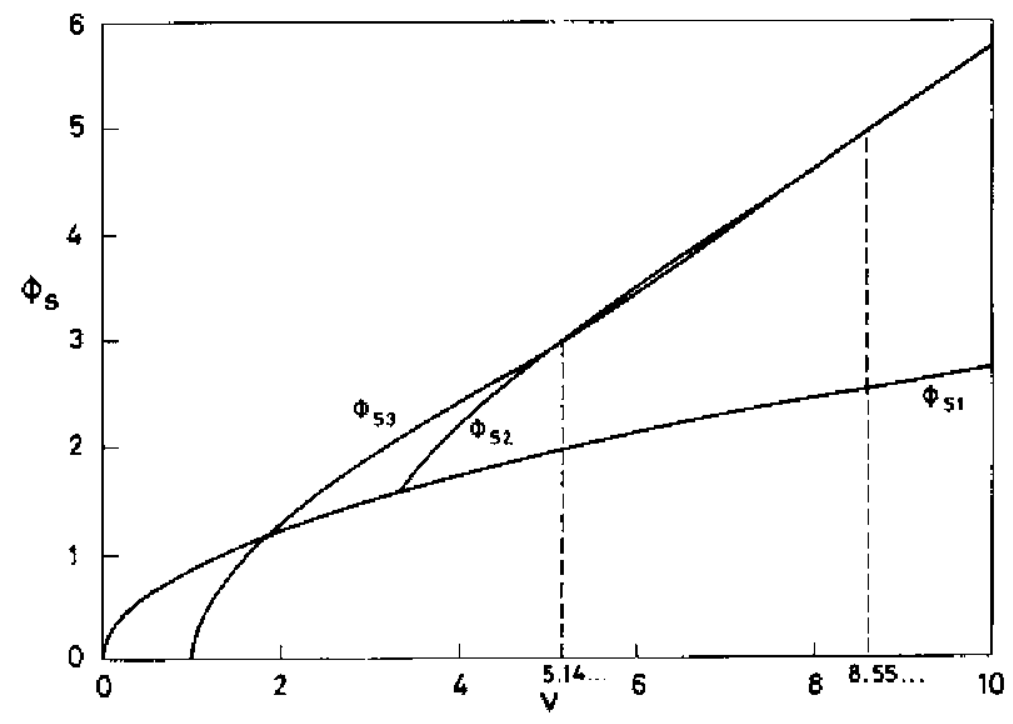

FJG. 5. The first coordinate of points 1,2 , and 3 in Fig. 4 in terms of $\nu$.

(ii) If $B>0$ and $l<\nu<\nu_{0}=1.8446 \cdots$, then the situation is as in one of the sketches (a) or (b) of Fig. 4. The curve $C_{2}^{\prime}$ intersect the curve $C_{1}$ (at $p_{3}$ ), but it does not necessarily intersect the curve $C_{2}$ (since $\Phi_{s 1}>\Phi_{s 3}$ and the curve $C_{2}$ is below the curve $C_{2}^{\prime}$ for sufficiently small $\boldsymbol{\Phi}_{s}$ ).

(iii) If $B>0$ and $\nu=\nu_{0}$, then $p_{1}$ and $p_{3}$ coalesce. At the resulting point, the linearized problem around the steady state has a double eigenvalue $\omega=0$, which is geometriçally simple, and a pair of complex conjugate simple eigenvalues, $\omega= \pm i \Omega$, 
with $\Omega \neq 0$. This is a codimension-three degeneracy that has been partially analyzed in [12], where it has been shown that it leads to bifurcation to two- and threedimensional tori. That degeneracy corresponds to the following situation: a Hopf bifurcation point crosses one of the bending points 1 or 2 of the response curve of Fig. 1 and, simultaneously, that bending point exhibits a saddle-node degeneracy.

(iv) If $B>0$ and $\nu_{0}<\nu \leqq 10 / 3$ (respectively if $10 / 3<\nu<\nu_{1}$ or, more generally, if $\nu_{2 k}<\nu<\nu_{2 k+1}$ for some $k \geqq 1$ ), then the situation is as in one of the sketches (c) or (d) (respectively, (e) or (f)) of Fig. 3. Therefore, if $\nu_{0}<\nu<\nu_{1}$, then the curves $C_{2}$ and $C_{2}^{\prime}$ intersect at (at least) a point $p_{4}$. At $p_{4}$ the linearized problem around the steady state possesses two pairs of complex conjugate simple eigenvalues $\omega= \pm i \Omega_{1}$ and $\omega= \pm i \Omega_{2}$, with $\Omega_{1} \neq 0$ and $\Omega_{2} \neq 0$. This is a codimension-two singularity whose universal unfolding has been completely analyzed [9]; in particular, it is known that the degeneracy leads to bifurcation to tori. The degeneracy corresponds to the following situation: two Hopf bifurcation points, with linearly independent eigenspaces, coalesce.

(v) If $B>0$ and $\nu=\nu_{1}$ (or, more generally, $\nu=\nu_{k}$ for some $k>1$ ), then the points $p_{2}$ and $p_{3}$ coalesce. At the resulting point, the linearized problem around the steady state has a simple zero eigenvalue $\omega=0$ and two pairs of complex conjugate simple eigenvalues $\omega= \pm i \Omega$, and $\omega= \pm i \Omega_{2}$, with $\Omega_{1} \neq 0$ and $\Omega_{2} \neq 0$. This is a codimension-three degeneracy that has been partially analyzed in [13] and leads to bifurcation to twoand three-dimensional tori. It corresponds to the following situation: two Hopf bifurcation points, of linearly independent eigenspaces, simultaneously cross one of the bending points 1 or 2 of the response curve of Fig. 1 .

(vi) If $B>0$ and $\nu_{2 k-1}<y<\nu_{2 k}$ for some $k \geqslant 1$, then the situation is as in one of the sketches $(\mathrm{g})$ or $(\mathrm{h})$ of Fig. 4 . Observe that the curves $C_{2}$ and $C_{2}^{\prime}$ intersect at a point $p_{4}$, where a degeneracy of codimension-two (namely, that appearing in case (iv)) takes place. Nevertheless, that degeneracy appears now at a point of the middle branch of the $S$-shaped part of the response curve of Fig. 1, where the linearized problem around the steady state has a real eigenvalue that is strictly positive. Therefore, no attractor of the dynamical system defined by (3.1)-(3.4) can bifurcate from the steady state near that singularity. Thus it is not of much interest.

To summarize the above results, the following degeneracies of codimensions two and three, leading to quasi-periodic behavior of $(3.1)-(3.4)$, have been found above $\left(\omega_{1}, \omega_{2}, \cdots\right.$ are the eigenvalues of the linearized problem around the steady state with zero real part):

(i) $\omega_{1}=0, \omega_{2,3}= \pm i \Omega$, with $\Omega \neq 0$;

(ii) $\omega_{1,2}= \pm i \Omega_{1}, \omega_{3,4}= \pm i \Omega_{2}$ with $\Omega_{1} \neq 0$ and $\Omega_{2} \neq 0$;

(iii) $\omega_{1}=0$ (algebraically triple, geometrically simple);

(iv) $\omega_{1}=0$ (algebraically double, geometrically simple), $\omega_{2,3}= \pm i \Omega$ with $\Omega \neq 0$;

(v) $\omega_{1}=0, \omega_{2,3}= \pm i \Omega_{1}, \omega_{4,5}= \pm i \Omega_{2}$, with $\Omega_{1} \neq 0$ and $\Omega_{2} \neq 0$.

To show that the dynamical system associated with (3.1)-(3.4) exhibits stable quasiperiodic behavior, in $\$ 4$ we make a bifurcation analysis of the singularity (i). Let us recall that the normal forms associated with the degeneracies (i) and (ii) may be reduced to a second-order autonomous system of ODEs; therefore nothing more interesting than quasi-periodic phenomena (which corresponds to periodic solutions of the above-mentioned system of ODEs) may be seen locally, via center manifold theory and normal form reduction. The normal forms associated with the degeneracies (iii)-(v), instead, consist of (or may be reduced to) a third-order system of ODEs, which might exhibit chaotic behavior. However, to our knowledge, that question (as well as some other significant ones, concerning the universal unfolding of the degeneracies (ii)-(v)) remains unsolved. 
Finally, observe that the stability analysis above, of the spatially one-dimensional problem (3.1)-(3.4), must be seen with some caution from the physical point of view, since the real world is three-dimensional. That analysis is expected to apply to the three-dimensional version of (3.1)-(3.4) in $\Omega=]-1,1\left[\times \Omega_{1}\right.$, where $\Omega_{1} \subset \mathbb{R}^{2}$ is a bounded domain, if the boundary conditions (3.2) are imposed at $\{-1,1\} \times \Omega_{1}$, Neumann boundary conditions are imposed at $]-1, I\left[\times \partial \Omega_{1}\right.$, and the domain $\Omega_{1}$ is sufficiently small; this should be seen by extending well-known results by Conway, Hoff, and Smoller [24]. This, however, is not true if the domain $\Omega_{1}$ is sufficiently large; in that case, we expect additional degeneracies of the linear stability problem, of codimension greater than three. In the limiting case in which $\Omega$ is an infinite slab, a new source of chaotic behavior appears, namely, the so-called phase turbulence [25]. We do not pursue this important question any further in this paper.

4. Quasi-periodic and other complex phenomena for model 2 in one dimension. In this section we analyze (via local bifurcation theory) the model (3.1)-(3.4) near a point of the parameter space $(\Phi, \gamma, \nu, B)=\left(\Phi_{0}, \gamma_{0}, v_{0}, B_{0}\right)$, where the linearized problem around a uniform steady state $\left(u_{s 0}, v_{s 0}\right)$ exhibits the degeneracy (i) encountered in $\$ 3$. Recall that this degeneracy corresponds to the interaction of a Hopf bifurcation point and a bending point of the response curye. The analysis will be made by reducing the problem to a three-dimensional center manifold and obtaining its normal form; then we use the general analysis of this normal form in [9]. Let us mention that simitar (but different) normal forms are obtained at the interaction of a Hopf bifurcation point and either a transcritical or a pitchfork steady bifurcation point; see [26], [27] and also [9, pp. 396 and 410$]$.

We take $\Phi_{0}, \gamma_{0}, \nu_{0}$, and $B_{0}$ such that (3.12)-(3.18) hold for some positive vatues of the real parameters $\Omega=\Omega_{0}$ and $\Phi_{s}=\Phi_{s 0}$, where $\Phi_{s 0}$ satisfies (3.9). Then the linearized problem around the steady state (3.8) has the simple eigenvalues $\omega=i \Omega_{0}, \omega=-i \Omega_{0}$, and $\omega=0$, with $\left(V, U_{+}, U_{-}\right)=\left(V_{1}, U_{1+}, U_{1-}\right),\left(\bar{V}_{1}, \bar{U}_{1+}, \bar{U}_{1-}\right)$, and $\left(V_{2}, U_{2+}, U_{2-}\right)$ as associated eigenvectors, where (here and below) overbars denote complex conjugation, and

$$
\begin{gathered}
V_{1}(x)=\cosh \left(\sqrt{i \Omega_{0}} x\right) / \cosh \left(\sqrt{i \Omega_{0}}\right), \quad V_{2}(x)=1 \quad \text { in }-1 \leqq x \leqq 1 \\
U_{1+}(\xi)=U_{1-}(\xi)=\gamma_{0} \nu_{0}^{2} \Phi_{s 0}^{2}\left[\exp \left(\sqrt{i \Omega_{0}} \xi\right)-\exp \left(\Phi_{s 0} \xi\right)\right] / i \Omega_{0}\left(\nu_{0}+B \Phi_{s 0}\right)^{2} \\
U_{2+}(\xi)=U_{2-}(\xi)=\gamma_{0} \nu_{0}^{2} \Phi_{s 0} \xi \exp \left(\Phi_{s 0} \xi\right) / 2\left(\nu_{0}+B_{0} \Phi_{s 0}\right)^{2} \quad \text { in }-\infty<\xi \leqq 0
\end{gathered}
$$

In addition, we assume that

$$
\Phi_{s 0} \neq v_{0} / B_{0}
$$

This condition and (3.12) mean that the steady state under consideration is, in fact, one of the bending points 1 or 2 of the response curve of Fig. 1. If (4.3) does not hold, but conditions $(3.9),(3.12),(3.17),(3.18)$ are still imposed, then the steady state bifurcation diagram exhibits the cusp degeneracy (which corresponds to coalescence of the bending points 1 and 2), and the local dynamic problem presents a degeneracy of codimension-three that will not be considered here.

Now let the parameters $\nu, B, \gamma$, and $\phi^{2}$ be close to $\nu_{0}, B_{0}, \gamma_{0}$, and $\Phi_{0}^{2}$, and let the parameters $\varepsilon=\left(\varepsilon_{1}, \varepsilon_{1}, \varepsilon_{3}\right) \in \mathbb{R}^{3}$ and $\delta \in \mathbb{R}$ be defined by

$$
\begin{gathered}
\nu=\nu_{0}+\varepsilon_{1}, \quad B=B_{0}+\varepsilon_{2}, \quad \gamma=2\left[\nu+B\left(\Phi_{s 0}+\varepsilon_{3}\right)\right]^{2} / \nu B\left(\Phi_{s 0}+\varepsilon_{3}\right), \\
\Phi^{2}=\delta+\left(\Phi_{s 0}+\varepsilon_{3}\right)^{2} \exp \left[-\gamma+\gamma \nu /\left(\nu+B \Phi_{s \cap}+B \varepsilon_{3}\right)\right] .
\end{gathered}
$$


Note that $\left(\nu, B, \gamma, \Phi^{2}\right) \rightarrow\left(\nu_{0}, B_{0}, \gamma_{0}, \Phi_{0}^{2}\right)$ as $|\varepsilon|^{2}+\delta^{2} \rightarrow 0$, and that the map $(\varepsilon, \delta) \rightarrow$ $\left(\nu, B, \gamma, \Phi^{2}\right)$ defined by (4.4) is one-to-one if $|\varepsilon|^{2}+\delta^{2}$ is sufficiently small (its Jacobian at $|\varepsilon|=\delta=0$ is different from zero according to condition (4.3)). The parameters $\varepsilon$ and $\delta$ are defined in such a way that if $\delta=0$ and $|\varepsilon|$ is sufficiently small, then (3.1)-(3.4) possess the steady state

$$
\begin{aligned}
& v_{s}(\varepsilon)=\left[v+B\left(\Phi_{s 0}+\varepsilon_{3}\right)\right] / \nu, \quad u_{s+}(\xi, \varepsilon) u_{s-}(\xi, \varepsilon)=\exp \left[\left(\Phi_{s 0}+\varepsilon_{3}\right) \xi\right] \\
& \text { in }-\infty<\xi<0 .
\end{aligned}
$$

Also, (i) $v_{s}, u_{s+}$, and $u_{s-}$ depend smoothly on $\varepsilon$; (ii) $\left(v_{s}, u_{s t}\right) \rightarrow\left(v_{s 0}, u_{s 0 \pm}\right)$ ( $\equiv$ the basic steady state that we are considering) as $\varepsilon \rightarrow 0$ and $\delta \rightarrow 0$; and (iii) the linearized problem around the steady state (4.5) possesses the eigenvalue $\omega=0$ (for all $\varepsilon$ such that $|\varepsilon|$ is sufficiently small).

Now, $\mathrm{if}|\varepsilon|^{2}+\delta^{2}$ is sufficiently small, we consider a three-dimensional center manifold of the phase space of $(3.1)-\langle 3.4), M_{(\delta, \varepsilon)}$, which is invariant under the semiflow defined by (3.1)-(3.4). $M_{(\hat{b, \varepsilon)})}$ is defined in a neighborhood of the steady state $\left(v_{s 0}, u_{s 0+}, u_{s 0}\right)$, and $M_{(0,0)}$ contains that steady state and is tangent (at the steady state) to the linear manifold spanned by the eigenvectors (of the linearized problem) associated with the eigenvalues $i \Omega_{0},-i \Omega_{0}$, and 0 . In addition, we require $M_{(0, E)}$ to contain the steady state (4.5) (for $|\varepsilon|$ sufficiently small). $M_{(\delta, e)}$ may be represented parametrically (through the parameters $\alpha \in \mathbb{C}$ and $\beta \in \mathbb{R})$ as

$$
\begin{gathered}
v(x)=v_{s}(\varepsilon)+\alpha V_{1}(x)+\bar{\alpha} \bar{V}_{1}(x)+\beta V_{2}(x)+\tilde{V}(x ; \alpha, \bar{\alpha}, \beta ; \delta, \varepsilon) \\
u_{\perp}(\xi)=u_{s \pm}(\xi, \varepsilon)+\alpha U_{1 \pm}(\xi)+\bar{\alpha} \bar{U}_{1 \pm}(\xi)+\beta U_{2 \pm}(\xi)+\tilde{U}_{ \pm}(\xi ; \alpha, \bar{\alpha}, \beta ; \delta, \varepsilon)
\end{gathered}
$$

Here $V_{j}, U_{j+}, U_{j-}$ (for $j=1$ and 2 ), $v_{s}, u_{s+}$, and $u_{s-}$ are as given by (4.1), (4.2), (4.5), and the functions $\tilde{V}[-1,1] \times \mathscr{B} \rightarrow \mathbb{R}$ and $\left.\left.\tilde{U}_{+}, \tilde{U}_{-} ;\right]-\infty, 0\right] \times \mathscr{B} \rightarrow \mathbb{R}$ (for some neighborhood $Q B$ of the origin of $\mathbb{C}^{2} \times \mathbb{R}^{5}$ ) depend smoothly on $x$ or $\xi_{3} \alpha_{R}, \alpha, \beta, \delta$, and $\varepsilon$, where

$$
\alpha_{R} \equiv \operatorname{Re} \alpha \text { and } \alpha_{j} \equiv \operatorname{Im} \alpha .
$$

In addition, $\tilde{V}, \tilde{U}_{+}$, and $\tilde{U}_{-}$satisfy the determinancy conditions

$$
\int_{-1}^{1} \tilde{V} V_{j}^{*} d x+\int_{-\infty}^{0} \tilde{U}_{+} U_{j+}^{*} d \xi+\int_{-\infty}^{0} \tilde{U}_{-} U_{j-}^{*} d \xi=0 \text { for } j=1 \text { and } 2
$$

where $\left(V_{1}^{*}, U_{1+}^{*}, U_{1-}^{*}\right)$ and $\left(V_{2}^{*}, U_{2+}^{*}, U_{2-}^{*}\right)$ are eigenvectors of the adjoint linearized problem associated with the eigenvalues $i \Omega_{0}$ and 0 , i.e.,

$$
\begin{aligned}
& V_{1}^{*}(x) \equiv\left(\Phi_{s 0}^{2}+i \Omega_{0}\right) \cosh \left(\sqrt{i \Omega_{0}} x\right), \quad V_{2}^{*}(x) \equiv 1 \quad \text { in }-1 \leqq x \leqq 1, \\
& U_{1+}^{*}(\xi) \equiv U_{1-}^{*}(\xi) \equiv B_{0} \Phi_{s 0}^{2} \cosh \left(\sqrt{i \Omega_{0}}\right)\left[1-\exp \left(\sqrt{\Phi_{s 0}^{2}+i \Omega_{0}} \xi\right)\right], \\
& U_{2+}^{*}(\xi) \equiv U_{2-}^{*}(\xi) \equiv B_{0}\left[1-\exp \left(\Phi_{s 0} \xi\right)\right] \quad \text { in }-\infty<\xi \leqq 0 .
\end{aligned}
$$

Also, the steady state (4.5) is assumed to correspond to $\alpha=0, \beta=0$ if $\delta=0$, i.e.,

$$
\tilde{V} \equiv 0, \tilde{U}_{+} \equiv \tilde{U}_{-} \equiv 0 \quad \text { if } \alpha=\beta=\delta=0 .
$$

As a consequence of conditions (4.9) and (4.12), it may be seen that the following tangency conditions hold:

$$
\begin{gathered}
\partial \tilde{V} / \partial \alpha=\partial \tilde{V} / \partial \bar{\alpha}=\partial \tilde{V} / \partial \beta=0, \\
\partial \tilde{U}_{ \pm} / \partial \alpha=\partial \tilde{U}_{ \pm} / \partial \bar{\alpha}=\partial \tilde{U}_{ \pm} / \partial \beta=0 \quad \text { if } \alpha=0, \beta=0, \text { and } \varepsilon=0,
\end{gathered}
$$

where $\partial / \partial \alpha$ and $\partial / \partial \bar{\alpha}$ are the formal $\left(\tilde{V}, \tilde{U}_{+}\right.$, and $\tilde{U}_{-}$are not necessarily holomorphic) partial derivative operators, defined by $(\operatorname{see}(4.8))$

$$
\partial / \partial \alpha \equiv\left(\partial / \partial \alpha_{R}-i \partial / \partial \alpha_{J}\right) / 2, \quad \partial / \partial \bar{\alpha} \equiv\left(\partial / \partial \alpha_{R}+i \partial / \partial \alpha_{I}\right) / 2
$$


Now the restriction of the semiflow defined by (3.1)-(3.4) to the center manifold may be described by a third-order real (when decomplexified) system of ODEs of the form

$$
d \alpha / d t=f(\alpha, \bar{\alpha}, \beta ; \delta, \varepsilon), \quad d \beta / d t=g(\alpha, \bar{\alpha}, \beta ; \delta, \varepsilon),
$$

for some functions, $f: \mathscr{B} \rightarrow \mathbb{C}$ and $g: \mathscr{B} \rightarrow \mathbb{R}$ (for some neighborhood $\mathscr{B}$ of the origin of $\mathbb{C}^{2} \times \mathbb{R}^{5}$ ) that depend smoothly on $\alpha_{R}, \alpha_{1}$ (see $\left.(4.8)\right), \beta, \delta$, and $\varepsilon$. These functions are determined (together with the functions $\tilde{V}, \tilde{U}_{+}$, and $\tilde{U}_{-}$defined above), at least locally, by the problem posed by (4.9), (4.12), and (4.15)-(4.18) below, which are readily obtained by imposing the invariance of the center manifold under the semiflow defined by (3.1)-(3.4):

$$
\begin{aligned}
& \partial^{2} \tilde{V} / \partial x^{2}=\left(V_{1}+\partial \tilde{V} / \partial \alpha\right) f+(1+\partial \tilde{V} / \partial \beta) g-i \Omega_{0} \alpha V_{1}+\text { c.c. } \quad \text { in }-1<x<1, \\
& \partial \tilde{V} / \partial x= \mp \alpha \sqrt{i \Omega_{0}} \tanh \sqrt{i \Omega_{0}} \pm \nu\left[-B \Phi_{s} / \nu-\alpha-\beta-\tilde{V}\right] \\
& \pm B \Phi^{2} \exp \left[\gamma-\gamma /\left(v_{s}+\alpha+\bar{\alpha}+\beta+\bar{v}\right)\right] \\
& \cdot \int_{-\infty}^{0}\left[u_{*}+\alpha U_{1 \pm}+\beta U_{2 \pm}+\hat{U}_{ \pm}\right] d \xi+\text { c.c. at } x= \pm 1
\end{aligned}
$$

$\partial^{2} \tilde{U}_{ \pm} / \partial \xi^{2}=\left(U_{1 \pm}+\partial \tilde{U}_{ \pm} / \partial \alpha\right) f+\left(U_{2 \pm}+\partial \tilde{U}_{ \pm} / \partial \beta\right) g-\alpha \partial^{2} U_{1 \pm} / \partial \xi^{2}-\beta \partial^{2} U_{2 \pm} / \partial \xi^{2}-\Phi_{s}^{2} u_{s}$

$$
\begin{array}{r}
+\Phi^{2}\left(u_{s}+\alpha U_{1 \pm}+\beta U_{2 \pm}+\tilde{U}_{ \pm}\right) \exp \left[\gamma-\gamma /\left(v_{s}+\alpha+\bar{a}+\beta+\tilde{V}_{ \pm}\right)\right]+\text {c.c. } \\
\text { in }-\infty<\xi \xi<0,
\end{array}
$$

Hexe $\tilde{V}_{ \pm}=\tilde{V}( \pm 1 ; \alpha, \bar{a}, \beta ; \delta, \varepsilon), \partial / \partial \alpha$ is the operator defined in (4.13) and c.c. denotes the complex conjugate.

The problem posed by (4.9), (4.12), (4.15)-(4.18) may be solved by (regular) perturbation techniques, by seeking the asymptotic expansions $(p, q, r, s=$ nonnegative integers)

$$
\begin{aligned}
f(\alpha, \bar{\alpha}, \beta ; \delta, \varepsilon) & =\sum_{p} \sum_{q+r+2=p} f_{q r s}^{p}(\varepsilon, \delta) \alpha^{q} \bar{\alpha}^{r} \beta^{2}, \\
g(\alpha, \bar{\alpha}, \beta ; \delta, \varepsilon) & =\sum_{p} \sum_{q+r+s=p} g_{q r s}^{p}(\varepsilon, \delta) \alpha^{q} \bar{\alpha}^{r} \beta^{s}, \\
\tilde{V}(x ; \alpha, \bar{\alpha}, \beta ; \delta, \varepsilon) & =\sum_{p} \sum_{q+r+s=p} \tilde{V}_{q r s}^{p}(x ; \delta, \varepsilon) \alpha^{q} \bar{\alpha}^{r} \beta^{s}, \\
\tilde{U}_{ \pm}(\xi, \alpha, \bar{\alpha}, \beta ; \delta, \varepsilon) & =\sum_{p} \sum_{q+r+s=p} \tilde{U}_{ \pm q r s}^{p}(x ; \delta, \varepsilon) \alpha^{4} \bar{\alpha}^{\prime} \beta^{s} .
\end{aligned}
$$

The coefficients are uniquely determined from the system of recursive linear problems that results when the expansions (4.19) are substituted into (4.9), (4.12), (4.15)-(4.18), and the coefficient of each monomial $\alpha^{4} \bar{\alpha}^{\prime} \beta^{s}$ is set to zero. Below, we consider all coefficients up to order $p=3$ in the expansions of $f$ and $g$, but we must explicitly compute only a first approximation of the following:

$$
\begin{aligned}
g_{000}^{0}(\delta, \varepsilon) & =A_{0} \delta+O\left(\delta^{2}+|\varepsilon|^{2}\right) \\
\operatorname{Re}\left[f_{100}^{1}(0, \varepsilon)\right] & =A_{1} \varepsilon_{1}+A_{2} \varepsilon_{2}+A_{3} \varepsilon_{3}+O\left(|\varepsilon|^{2}\right) \\
\operatorname{Im}\left[f_{100}^{1}(0,0)\right] & =\Omega_{0,}, \\
\operatorname{Re}\left[f_{101}^{2}(0,0)\right] & =A_{4}, \quad g_{130}^{2}(0,0)=A_{5}, \quad g_{002}^{2}(0,0)=A_{6,}
\end{aligned}
$$

where the constants $A_{0}, \cdots, A_{6}$ are given in the Appendix $B$.

Now a third-order normal form of system (4.14) may be obtained as follows. We first truncate the expansions (4.19) of $f$ and $g$ by ignoring all terms of order $p \equiv 4$ and 
replace the resulting polynomial in (4.14). Then we consider a near-identity change of variables $(\alpha, \beta) \rightarrow(y\langle\alpha, \beta ; \delta, \varepsilon), z(\alpha, \beta ; \delta, \varepsilon)) \in \mathbb{C} \times \mathbb{R}$, with $y \simeq \alpha$ and $z \approx \beta$ as $(\alpha, \beta, \delta, \varepsilon) \rightarrow(0,0,0,0)$, of the form

$$
y=\sum_{p=0}^{3} \sum_{q+r+s a p} Y_{q r s}^{p}(\delta, \varepsilon) \alpha^{q} \bar{\alpha}^{r} \beta^{s}, \quad z=\sum_{p=0}^{3} \sum_{q+r+s=p} Z_{q r s}^{p}(\delta, \varepsilon) \alpha^{q} \bar{\alpha}^{r} \beta^{s},
$$

whose coefficients are selected in such a way that, in the new variables, system (4.14) becomes of the form

$$
\begin{aligned}
d y / d t=\left[a_{0}(\delta, \varepsilon)+i c_{0}(\delta, \varepsilon)\right] y+\left[a_{1}(\delta, \varepsilon)+i c_{1}(\delta, \varepsilon)\right] y z \\
+\left[a_{2}(\delta, \varepsilon)+i c_{2}(\delta, \varepsilon)\right] y^{2} \bar{y}+\left[a_{3}(\delta, \varepsilon)+i c_{3}(\delta, \varepsilon)\right] y z^{2}+O\left(|y|^{2}+z^{2}\right)^{2}, \\
d z / d t=b_{0}(\delta, \varepsilon)+b_{1}(\delta, \varepsilon) y \bar{y}+b_{2}(\delta, \varepsilon) z^{2}+b_{3}(\delta, \varepsilon) y \bar{y} z \\
+b_{4}(\delta, \varepsilon) z^{3}+O\left(|y|^{2}+z^{2}\right)^{2}
\end{aligned}
$$

where the real coefficients $a_{j}, b_{j}$, and $c_{j}$ are such that

$$
\begin{gathered}
b_{0}(\delta, \varepsilon)=A_{0} \delta+O\left(|\varepsilon|^{2}+\delta^{2}\right), \quad a_{0}(0, \varepsilon)=A_{1} \varepsilon_{1}+A_{2} \varepsilon_{2}+A_{3} \varepsilon_{3}+O\left(|\varepsilon|^{2}\right), \\
c_{0}(0,0)=\Omega_{0}, \quad a_{1}(0,0)+i c_{1}(0,0)=A_{4}, \quad b_{1}(0,0)=A_{5}, \quad b_{2}(0,0)=A_{6} .
\end{gathered}
$$

The terms appearing in the right-hand side of (4.22) cannot be annihilated by a change of variables of the type (4.21), and are called resonants.

That change of variables is seen to exist by applying the implicit function theorem to the system of equations that results when (4.21) is substituted into (4.14), and the coefficient of the monomials not appearing in (4.22) is set to zero (observe that $g_{002}^{2}(0,0) \neq 0$; see $(4.3)$ and $\left.(4.20)\right)$.

Now, when the new real variables $r>0$ and $\theta$, defined by $y=r \exp (i \theta)$ are introduced, (4.22) is written in the form

$$
\begin{aligned}
& d r / d t=a_{0} r+a_{1} r z+a_{2} r^{3}+a_{3} r z^{2}+O\left(r^{2}+z^{2}\right), \\
& d z / d t=b_{0}+b_{1} r^{2}+b_{2} z^{2}+b_{3} r^{2} z+b_{4} z^{3}+O\left(r^{2}+z^{2}\right)^{2}, \\
& d \theta / d t=c_{0}+c_{1} z+c_{2} r^{2}+c_{3} z^{2}+O\left(r^{2}+z^{2}\right)^{4} / r .
\end{aligned}
$$

Then (4.25), (4.26) are decoupled from (4.27), and they may be solved separately. Note that the stationary (respectively, periodic) solutions of (4.25), (4.26) with $r>0$ are periodic (respectively, quasi-periodic) solutions of (4.25)-(4.27). Now we may use the fairly complete analysis of (4.25), (4.26) made in [9, pp. 376-396]. To this end, we first reduce $(4.23),(4.25)$ to standard form by using the new variables and parameters

$$
\begin{gathered}
u=\left|b_{1} / b_{2}\right|^{1 / 2} r, \quad v=-b_{2} z, \quad \mu_{1}=a_{0}, \quad \mu_{2}=-b_{0} b_{2}, \quad a=-a_{1} / b_{2}, \\
b=-\operatorname{sign}\left[b_{2} / b_{1}\right](= \pm 1), \quad c=a_{2} b_{2}^{2} / b_{1}^{2}, \quad d=a_{3} / b_{2}^{2}, \\
e=b_{2}^{2} b_{3} / b_{1}^{2}, \quad f=b_{4} / b_{2}^{2}
\end{gathered}
$$

to write $(4.25),(4.26)$ as

$$
\begin{aligned}
& d u / d t=\mu_{1} u+a u v+c u^{3}+d u v^{2}+O\left(u^{2}+v^{2}\right)^{2}, \\
& d v / d t=\mu_{2}+b u^{2}-v^{2}+e u^{2} v+f v^{3}+O\left(u^{2}+v^{2}\right)^{2} .
\end{aligned}
$$

Here we assume that $b_{1} \neq 0$ (note that $b_{2} \neq 0$; see (4.3), (4.20)).

Now, when using (4.24), (4.28) and the expressions for $A_{0}, A_{1}, A_{2}$, and $A_{3}$ in Appendix $\mathrm{B}$, it is seen that the map $(\delta, \varepsilon) \rightarrow\left(\mu_{1}(\delta, \varepsilon), \mu_{2}(\delta, \varepsilon)\right)$ of $\mathbb{R}^{4}$ into $\mathbb{R}^{2}$ is such that the rank of its Jacobian matrix at $(\delta, \varepsilon)=(0,0)$ is always equal to 2 . As a consequence, any point $\left(\mu_{1}, \mu_{2}\right)$ in a whole neighborhood of the origin of $\mathbb{R}^{2}$ may be reached if $\delta$ and $\varepsilon$ are appropriately chosen. 
From the analysis in [9], if $a<0$ and $b=1$, then system (4.29) exhibits a Hopf bifurcation at a curve of the plane $\mu_{1}-\mu_{2}$ (for fixed values of the remaining parameters) of the form $\mu_{1}=\mu_{1}\left(\mu_{2}\right)$; with $\mu_{2}<0$, such that $\mu_{1}^{\prime}(0)=0$. At that curve, the original problem exhibits a bifurcation to torus; it is well known (see, e.g., [10, pp. 292-313]) that, when having at least two free parameters (we have four of them available), near that bifurcation the dynamical system exhibits a quite complex dynamic behavior, which includes a large number of periodic solutions and, quite frequently, chaotic behavior. Also, if $a>0$ and $b=-1$, then near a curve of the plane $\mu_{1}-\mu_{2}$, of the type $\mu_{1}=\mu_{1}\left(\mu_{2}\right)$, with $\mu_{2}>0$, system (4.29) presents a homoclinic bifurcation that is expected to yield also quite complex dynamic behavior for the original system (see, e.g., [9, p. 394]). Both situations $(a<0, b=1$ and $a>0, b=-1)$ do occur for appropriate values of the parameters in our case, as it comes from the plot in Fig. 6, where the curves $\operatorname{Re}\left(A_{4}\right)=0, A_{5}=0$, and $A_{6}=0$ are represented in terms of $B$ and $\nu$ (the expressions of $A_{4}, A_{5}$, and $A_{6}$ in Appendix $B$ depend on the parameters $\Omega, \phi_{s}, B$, and $\nu$, but $\phi_{s}$ and $\Omega$ are eliminated when using (3.17), (3.18)). These curves define several regions in the plane $\nu-B$. In particular, in region $I, \operatorname{Re}\left(A_{4}\right)>0, A_{5}<0, A_{6}>0$, and (see (4.24), (4.28)) the parameters $a$ and $b$ are such that $a<0$ and $b=1$; similarly, in region $\mathrm{II}, \operatorname{Re}\left(A_{4}\right)<0, A_{5}<0, A_{6}<0, a>0$, and $b=-1$. Note that a blow-up of the region near the point $\nu=10 / 3, B=2 \sqrt{10} / 3$ is necessary to appreciate a second point of intersection: of the curves $\operatorname{Re}\left(A_{4}\right)=0$ and $A_{5}=0$.

5. Conclusions. We have obtained two realistic submodels of (1.1), (1.2) in the limit (1.3) for $\nu \sim 1$, which we call models 1 and 2. Their formal derivation by singular perturbation techniques, was made in Appendix $A$ and $\$ 2$.

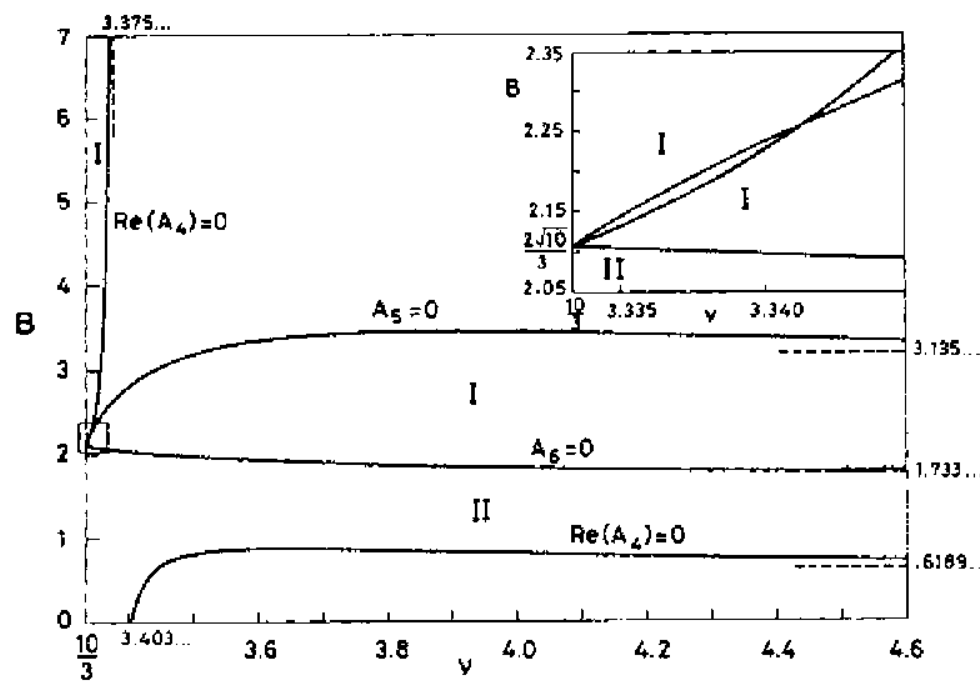

FIG. 6. The curves $\operatorname{Re}\left(A_{4}\right)=0, A_{5}=0$, and $A_{6}=0$.

Model 1 has been already considered in the literature, as explained in $\$ 2.1$. Model 2 , instead, is new (to our knowledge), and it is quite interesting from the dynamical point of view. The steady states of model 2 and their linear stability were analyzed in $\$ 4$, where we showed that the model presents several degeneracies of codimensions two and three, which are likely to lead to chaotic behavior. One of these degeneracies was analyzed in detail in $\$ 4$, where we showed that, for appropriate values of the 
parameters, the dynamics of the model is quite rich. Those results lead us to the conjecture that model 2 should present chaotic behavior for approprate values of the parameters.

Since, as we explained in 1 , model 2 is obtained in a realistic limit, we expect that the results in this paper explain some experimentally observed, quite rich dynamic Gehavior under apparently isothermal conditions; see, e.g., [28], [29], and [1, Vol. II, pp. 140-141]. Finally, to our knowledge, model 2 is the first submodel of (1.1), (1.2) in the literature such that (a) it is obtained for realistic limiting values of the parameters, (b) it is simpler than (1.1), (1.2), and (c) it exhibits generically stable quasi-periodic behavior.

Appendix A. Asymptotic derivation of the basic model. In this appendix we consider the limit (1.5) with $\nu \sim 1$, for the problem (1.1), (1.2), with initial conditions

$$
u(x, 0)=\tilde{u}(x)>0, \quad v(x, 0)=\tilde{v}(x)>0 \quad \text { in } \Omega,
$$

where the smooth functions $\tilde{u}$ and $\tilde{v}$ satisfy the boundary conditions at $\partial \Omega$. For brevity, only the spatiatly three-dimensional case is considered, but the results of this section are also valid in one and two space dimensions. Also, for simplicity in the presentation, we assume that $\gamma \sim O(1)$. Again, it may be seen that the basic model applies also if $\gamma \gg 1$.

Below, we encounter two thin boundary layers beside the boundary of $\Omega$. To describe the (inner) solution in these layers, we introduce, in a neighborhood $B$ of each point of $\partial \Omega$, a curvilinear coordinate system $\left(\eta_{,} \eta_{1}, \eta_{2}\right)$ such that $\partial \Omega$ is the parametric surface $\eta=0$ and $\eta$ is a coordinate along the outward unit normal to $\partial \Omega$. Then the Laplacian operator is written as

$$
\Delta=\partial^{2} / \partial \eta^{2}+h^{i j} \partial^{2} / \partial \eta^{i} \partial \eta^{j}+p \partial / \partial \eta+p^{k} \partial / \partial \eta^{k},
$$

where $h^{i j}, p$, and $p^{k}(i, j, k=1$ and 2$)$ are appropriate smooth functions defined in the neighborhood $B$.

Two timescales must be considered. For $t \sim 1 / \sigma$, a boundary layer of thickness $1 / \sqrt{\sigma}$ appears beside the boundary of the domain. We use the time variable $r=\sigma t$ and the inner variables $\xi=\sqrt{\sigma} \eta, \eta^{2}$, and $\eta^{3}$ in the boundary layer, and we seek the expansions

$$
u=u_{0}+u_{1} / \sqrt{\sigma}+\cdots, \quad v=v_{0}+v_{1} / \sqrt{\sigma}+\cdots
$$

in the outer zone, and of the form

$$
u=\bar{u}_{0}+\bar{u}_{1} / \sqrt{\sigma}+\cdots, \quad v=\bar{v}_{0}+\bar{v}_{1} / \sqrt{\sigma}+\cdots
$$

in the boundary layer. Then $u_{0}$ and $v_{0}$ are given by

$$
\begin{gathered}
\lambda \partial u_{0} / \partial \tau=0, \quad u_{0}(x, y, z ; 0)=\tilde{u}(x, y, z), \\
\partial v_{0} / \partial \tau=b \varphi^{2} u_{0} \exp \left(y-\gamma / v_{0}\right), \quad v_{v}(x, y, z ; 0)=\tilde{v}(x, y, z),
\end{gathered}
$$

and $\bar{u}_{0}$ and $\bar{v}_{0}$ are given by

$$
\begin{gathered}
\lambda \partial \bar{u}_{0} / \partial \tau=0, \quad \bar{u}_{0}\left(\xi, \eta^{\mathrm{l}}, \eta^{2} ; 0\right)=\bar{u}\left(0, \eta^{1}, \eta^{2}\right), \\
\partial \bar{v}_{0} / \partial \tau=\partial^{2} \bar{v}_{0} / \partial \xi^{2}+b \varphi^{2} \bar{u}_{0} \exp \left(\gamma-\gamma / v_{0}\right), \partial \bar{v}_{0} / \partial \xi=0 \quad \text { at } \xi=0, \\
\bar{v}_{0}\left(\xi, \eta^{1}, \eta^{2} ; 0\right)=\bar{v}\left(0, \eta^{1}, \eta^{2}\right),
\end{gathered}
$$

where the parameters $\lambda, b$, and $\varphi^{2}$ are as defined in (1.11). By uniqueness of solution of (A.4)-(A.6), we obtain that neither $\bar{u}_{0}$ nor $\bar{v}_{0}$ depend on $\xi$. Then $\bar{v}_{0}$ is given by

$$
\partial \bar{v}_{0} / \partial \tau=b \varphi^{2} \bar{u}_{0} \exp \left(\gamma-\gamma / v_{0}\right),
$$


and, to the leading order, the boundary layer can be ignored at this time stage, since the inner problem (A.4), (A.6), (A.7) is obtained by witing the outer problem in the inner variables. This is no longer true for higher-order terms, since, for example, $\bar{v}^{1}$ does depend on $\xi$ for $\tau>0$, as is easily seen.

The behavior of $u_{0}$ and $v_{0}$ as $\tau \rightarrow \infty$ is easily obtained, from (A.2)-(A.3), to be

$$
u_{0}=\tilde{u}, \quad \bar{v}_{0}=b \varphi^{2} \tilde{u} \exp (\gamma)_{\tau}+O(t) \quad \text { in } \Omega .
$$

Therefore $v_{0} \sim \sigma$ as $r \sim \sigma$, and we are led to the following time stage for $t \sim 1$. Now a new boundary layer of thickness $1 / \sigma$ appears beside $\partial \Omega$. We use the inner variables $\xi=\eta \sigma, \eta^{1}$, and $\eta^{2}$ in this new boundary layer, and seek expansions of the form

$$
u=u_{0}+u_{1} / \sqrt{\sigma}+\cdots \quad v=\sigma V_{0}+\sqrt{\sigma} V_{1}+\cdots
$$

in the outer zone, of the form

$$
u=\bar{u}_{0}+\bar{u}_{1} / \sqrt{\sigma}+\cdots, \quad v=\sigma \bar{V}_{0}+\sqrt{\sigma} \bar{V}_{1}+\cdots
$$

in the first boundary layer (of thickness $1 / \sqrt{\sigma}$ ), and of the form

$$
u=\overline{\bar{u}}_{0}+\overline{\bar{u}}_{1} / \sqrt{\sigma}+\overline{\bar{u}}_{2} / \sigma+\cdots, \quad v=\sigma \overline{\bar{V}}_{0}+\sqrt{\sigma} \overline{\bar{V}}_{1}+\overline{\bar{V}}_{2}+\cdots
$$

in the second boundary layer (of thickness $1 / \sigma$ ). Then $u_{0}$ and $V_{0}$ are given by

$$
\begin{aligned}
& \lambda \partial u_{0} / \partial t=-\varphi^{2} u_{0} \exp (\gamma), \\
& \partial V_{0} / \partial t=\Delta V_{0}+b \varphi^{2} u_{0} \exp (\gamma)
\end{aligned}
$$

$\bar{u}_{0}, \bar{V}_{0}$, and $\bar{V}_{1}$ are given by

$$
\begin{array}{ll}
\text { (A.10) } & \lambda \partial \bar{u}_{0} / \partial t=-\varphi^{2} \bar{u}_{0} \exp (\gamma), \\
\text { (A.11) } & \partial^{2} \bar{V}_{0} / \partial \xi^{2}=\partial^{2} \bar{V}_{1} / \partial \xi^{2}=0 ;
\end{array}
$$

and $\overline{\bar{u}}_{u}, \overline{\bar{V}}_{0}, \overline{\bar{V}}_{1}$, and $\overline{\bar{V}}_{2}$ are given by

$$
\begin{gathered}
\lambda \partial \overline{\bar{u}}_{0} / \partial t=\partial^{2} \overline{\bar{u}}_{0} / \partial \zeta^{2}-\varphi^{2} \overline{\bar{u}}_{0} \exp (\gamma) ; \quad \partial \overline{\bar{u}}_{0} / \partial \zeta=1-\overline{\bar{u}}_{0} \quad \text { at } \zeta=0, \\
\partial^{2} \overline{\bar{V}}_{0} / \partial \zeta^{2}=\partial^{2} \overline{\bar{V}}_{1} / \partial \zeta^{2}=0 ; \quad \partial \overline{\bar{V}}_{0} / \partial \zeta=\partial \overline{\bar{V}}_{1} / \partial \zeta=0 \quad \text { at } \zeta=0, \\
\partial^{2} \bar{V}_{2} / \partial \zeta^{2}=0 ; \quad \partial \overline{\bar{V}}_{2} / \partial \zeta=-\nu \overline{\bar{V}}_{0} \quad \text { at } \zeta=0 .
\end{gathered}
$$

We do not write the initial conditions for the above problem, which come from matching conditions with the earlier time stage. From (A.13), we obtain that neither $\overline{\bar{V}}_{0}$ nor $\overline{\bar{V}}_{1}$ depend on $\zeta$, and (A.14) yields $\partial \bar{V}_{2} / \partial \zeta=-\nu \overline{\bar{V}}_{0}$ in $-\infty<\zeta<0$. Then, from (A.11) and matching conditions between the two boundary layers, we obtain that $\bar{V}_{0}$ does not depend on $\xi$, and $\partial \bar{V}_{2} / \partial \xi=-\nu \bar{V}_{0}$ in $-\infty<\xi<0$. Finally, matching conditions between the outer zone and the first boundary layer yield

$$
\partial V_{0} / \partial n=-\nu V_{0} \text { at } \partial \Omega \text {. }
$$

Now, from (A.8) and (A.10), we obtain that $u_{0} \rightarrow 0, \bar{u}_{0} \rightarrow 0$ as $t \rightarrow \infty$, and then (A.9) and (A.15) yield $V_{0} \rightarrow 0$ as $t \rightarrow \infty$. In the same way, from the equations giving higher-order terms, which are not written for brevity, we obtain that, as $t \rightarrow \infty, v=O(1)$ everywhere in $\Omega$, and $u=o(1 / \sigma)$ in the outer zone and in the first boundary layer. Therefore, for sufficiently large $t$,

$$
u=o(1 / \sigma), \quad \partial v / \partial t=\Delta v+o(1)
$$

both in the outer zone and in the first boundary layer, and these two zones need not be distinguished in frst approximation. Then, if we seek the expansions $v=v_{0}+v_{1} / \sqrt{\sigma}+\cdots$, in the outer zone, and

$$
u=\overline{\bar{u}}_{0}+\overline{\bar{u}}_{1} / \sqrt{\sigma}+\cdots, \quad v=\bar{v}_{0}+\bar{v}_{1} / \sqrt{\sigma}+\bar{v}_{2} / \sigma+\cdots
$$


in the second boundary layer, $v_{0}$ satisfies $(1.7), \bar{u}_{0}$ satisfies $(1.9),(1.10)$, and $\bar{v}_{0}, \bar{v}_{1}$, and $\overline{\bar{v}}_{2}$ are given by

$$
\begin{gathered}
\partial^{2} \bar{v}_{0} / \partial \zeta^{2}=\partial^{2} \bar{v}_{1} / \partial \zeta^{2}=0 ; \quad \partial \bar{v}_{0} / \partial \zeta=\partial \bar{v}_{1} / \partial \zeta=0 \quad \text { at } \zeta=0, \\
0=\partial^{2} \overline{\bar{v}}_{2} / \partial \zeta^{2}+b \varphi^{2} \overline{\bar{u}}_{0} \exp \left(\gamma-\gamma / \overline{\bar{v}}_{0}\right), \quad \partial \overline{\bar{v}}_{2} / \partial \zeta=\nu\left(1-\bar{v}_{0}\right) \quad \text { at } \zeta=0 .
\end{gathered}
$$

Then neither $\overline{\bar{v}}_{0}$ nor $\overline{\bar{v}}_{1}$ depend on $\zeta$, and, by integrating (A.16) from $-\infty$ to 0 , we obtain that

$$
\partial \overline{\bar{v}}_{2} / \partial \zeta=\nu\left(1-\overline{\bar{v}}_{0}\right)+b \varphi^{2} \exp \left(\gamma-\gamma / \overline{\bar{v}}_{0}\right) \int_{-\infty}^{0} \overline{\bar{u}}_{0}(\zeta, \tau) d \zeta \quad \text { as } \zeta \rightarrow-\infty
$$

Then matching conditions between the outer zone and the boundary layer show that $v_{0}$ satisfies (1.8) at $2 \Omega$, and the derivation of $(1.7)-(1.10)$ is complete.

Finally, observe that if the nonlinearity $\phi^{2} u \exp (\gamma-\gamma / v)$ is replaced in (1.1), (1.2) by a more general one, $\phi^{2} f(u, v)$, where $f$ is a nonnegative smooth function such that $f(0, v) \equiv 0$, then the above analysis stands after obvious changes. The resulting model is that obtained from model 1 by replacing the terms $\varphi^{2} \exp (\gamma-\gamma / v)$ $\int_{-\infty}^{0} u(\zeta, t) d \zeta$ and $\varphi^{2} u \exp (\gamma-\gamma / v)$ by $\varphi^{2} \int_{-\infty}^{\theta} f(u(\zeta, t), v(t)) d \zeta$ and $\varphi^{2} f(u, v)$ in $(1.7)$ and $(1.8)$, respectively.

Appendix B. The values of the constants $A_{0}, A_{1}, \cdots A_{6}$ that appear in (4.20) are as follows:

$$
\begin{aligned}
& A_{0}=\frac{2 B \Phi_{s}^{3}}{\Phi^{2}\left(4 \Phi_{s}^{2}-3 \nu\right)}>0, \\
& A_{1}=\frac{-7 B \Phi_{s}-3 \nu+\frac{4}{\nu}\left(3 B \Phi_{s}+\nu\right) \sqrt{i \Omega} \tanh \sqrt{i \Omega}}{\left(B \Phi_{s}+\nu\right) C_{1}} \\
& A_{2}=\frac{-2\left(B \Phi_{s}-\nu\left(1+2 \Phi_{s}\right)\right)+\frac{2 \nu\left(B \Phi_{s}+\nu\right)}{i \Omega B}\left(\frac{\Phi_{s}^{3}}{\sqrt{\Phi_{s}^{2}-i \Omega}}-\left(\Phi_{s}^{2}-i \Omega\right)\right)+\frac{4}{\nu} \sqrt{i \Omega} \tanh \sqrt{i \Omega}}{\left(B \Phi_{s}+\nu\right) C_{1}} \\
& A_{3}=\frac{2\left(\nu(1+2 B)-B \Phi_{s}\right)+\left(\frac{\left(8 i \Omega+2 \Phi_{s}^{2}\right)\left(B \Phi_{s}+\nu\right)}{\Phi_{s} i \Omega}+4 B \Phi_{s}-\nu(1+2 B)\right) \sqrt{i \Omega} \tanh \sqrt{i \Omega}}{\left(B \Phi_{s}+\nu\right) C_{1}} \\
& +\frac{\frac{4 \nu \Phi_{s}\left(B \Phi_{s}+\nu\right)}{i \Omega}\left(\frac{\Phi_{s}}{\sqrt{\Phi_{s}^{2}+i \Omega}}-1\right)-2 \Phi_{s}\left(B \Phi_{s}+\nu\right) C_{2}}{\left(B \Phi_{s}+\nu\right) C_{1}}-\frac{6 \nu}{\Phi_{s} C_{1}} \\
& A_{4}=\frac{\frac{2 \nu^{3}}{B \Phi_{s}}+\left(\frac{2\left(\Phi_{s}^{2}+i \Omega\right)\left(B \Phi_{s}+\nu\right) \nu}{B \Phi_{s}}-2 i \Omega \nu\right) \frac{\tanh \sqrt{i \Omega}}{\sqrt{i \Omega}}}{\left(B \Phi_{s}+\nu\right) C_{1}} \\
& -\frac{2 y^{2}}{B \Phi_{s} C_{1}}\left[\frac{\left(2 \Omega\left(\Phi_{s}+\sqrt{\Phi_{s}^{2}+i \Omega}\right) i+\Phi_{s}^{3}\right) \Phi_{s}^{2}}{\left(\Phi_{s}^{2}+i \Omega\right)^{3 / 2}\left(\Phi_{s}+\sqrt{\Phi_{s}^{2}+i \Omega}\right)^{2}}+1\right]
\end{aligned}
$$


$A_{5}=\frac{4 \Phi_{s} \nu\left(\frac{2 \nu^{2}}{\nu+B \Phi_{s}}+\frac{4 \nu \Phi_{s}^{3}}{\Omega}\left[\frac{-b}{\sqrt{\Phi_{s}^{4}+\Omega^{2}}}\right]+2 \Phi_{s}^{2}\left(\frac{\sqrt{2 \Omega}}{\Omega} \frac{(\sinh \sqrt{2 \Omega}+\sin \sqrt{2 \bar{\Omega}})}{(\cosh \sqrt{2 \Omega}+\cos \sqrt{2 \bar{\Omega}})}\right)\right)}{B\left(4 \Phi_{s}^{2}-3 \nu\right)}$

$A_{6}=\frac{4 \Phi_{s} \nu^{2}\left(B \Phi_{s}-\nu\right)}{B\left(B \Phi_{s}+\nu\right)\left(4 \Phi_{s}^{2}-3 \nu\right)}$,

where

$$
\begin{aligned}
& C_{1}=\frac{1}{2}\left(\frac{\tanh \sqrt{i \Omega}}{\sqrt{i \Omega}}+\frac{2}{1+\cosh 2 \sqrt{i \Omega}}\right)-\frac{1, \Phi_{s}^{2}}{\left(\Phi_{s}^{2}+i \Omega\right)^{3 / 2}}\left[\frac{\Phi_{s}+2 \sqrt{\Phi_{s}^{2}+i \Omega}}{\left(\Phi_{s}+\sqrt{\Phi_{s}^{2}+i \Omega}\right)^{2}}\right], \\
& C_{2}=\frac{\nu \Phi_{s}^{2}}{\left(\Phi_{s}^{2}+i \Omega\right)^{3 / 2}}\left[\frac{\Phi_{s}+2 \sqrt{\Phi_{s}^{2}+i \Omega}}{\left(\Phi_{s}+\sqrt{\Phi_{s}^{2}+i \Omega}\right)^{2}}\right] .
\end{aligned}
$$

Here the suffix 0 has been dropped out everywhere from the parameters $B_{0}, \nu_{0}$, $\phi_{s 0}$, and $\Omega_{0}$, which are defined at the beginning of $\S 4$ and $b$ is defined in (3.15).

\section{REFERENCES}

[1] R. ARJs, The Mathematical Theory of Diffision and Reaction in Permeable Catalysts, Vols. I and II, Clarendon Press, Oxford, UK, 1975.

[2] N. R. AMUNDSON AND L. R. RAYMOND, Stability in distributed parameter systems, Amer. Inst. Chem. Engtg., Il (1965), pp. 339-350.

[3] I. E. PARRA AND J. M. VEOA, Local nonlinear stability of the steady states in an isothermal catalyst, SIAM J. Appl. Math., 48 (1988), pp. 854-881.

[4] J. M. VEGA, Invariant regions and global asymptotic stobility in an isothermal catalyst, SIAM J. Math. Anal., 19 (1988), pp. 774-796.

[5] R. Hu, V, Balakotaiah, and D. Luss, Multiplicity features of porous catalytic pellets. Part I: Single zeroth-order reaction case, Chem. Engrg. Sci., 40 (1985), pp. 589-598; Part II; Influence of reaction order and pellet geometry, Chem. Engrg. Sci., 40 (1985), pp. 599-610, Part III: Uni(gueness criteria for the lumped thermal model, Chem. Engrg. Sci,, 41 (1986), pp. 1525-1537.

[6] P. L. LIONs, On the existence of positive solutions of semilinear elliptic equations, S1AM Rev., 24 (1982), pp. $41-467$.

[7] J. K. Hale, Asymptotic Behavior of Dissipative Systems, American Mathematical Society, Providence, RI, 1988.

[8] L. M. PIs'MEN AND Y. I. KHARKATS, Asymmetric state of a heterogeneous exothermic reaction, Dokl. Akad Sci. USSR, Chemical Technology Section, 178/179 (1968).

[9] J. GuCKenhelMer AND P. Holmes, Nonlinear Oscillations, Dynamical Systems and Bifurcations of Vector Fields, Springer-Verlag, Berlin, New York, 1983.

[10] V. I. ARNoLv, Geometrical Methods in the Theory of Ordinary Differential Equations, Springer-Verlag, Berlin, New York, 1983.

[11] P. YU AND K. HUSEYIN, Bifurcations associated with a three-fold zero eigenvalue, Quart. App]. Math., 46 (1988), pp. 193-216.

[12] - Bifurcation associaled with a double zero and a pair of pure imaginary eigenvalues, SIAM $j$. Appl. Math., 48 (1988), pp. 229-261.

[13] K. HUSEY'N ANU P. YU, Bifurcation associated with a simple zero and two pairs of pure imaginary eigenvalues, in CMS/AMS Proc. Internat. Conf. on Oscillation, Bifurcation and Chaos, Toronto, Ontário, Canada, 8 (1986), pp. 465-484.

[14] L. M. PIS'MEN, Methods of singularity theory in the andysis of dynamics of reactive systemts, in Lecture Notes in Appl. Math., Vol. 24-II, American Mathematical Society, Providence, RI, 1986.

[15] J. B. PLANEALX, K. F. JENSEN, ANU W. W. FARR, Dymamic behavior of contintous stirred-tank reactors with extraneous thermal capacitance, in Lecture Notes in Appl. Math., Vol. 24-II, American Mathematical Society, Providence, RI, 1986. 
[16] D. LuSs, J. E. BAJLEY, AND S. SHARMA, Asymmetric steady states in cotalytic slabs with uniform and non-uriform environment, Chem. Engrg. Sci, 27 (1972), pp. 1555-1567.

[17] D. G. ARONSON AND L. A. PELET]ER, Global stability of symmetric and asymmetric concentration profiles in catalyic particles, Arch. Rat. Mech. Anal., 54 (1974), pp. 175-204.

[18] D. G. ARonson, A comparison method for stability analysis of nonlinear parabalic problems, SIAM Rev., 20 (1978), pp. 245-264.

[19] J. M. BALl AND L. A. PELETIER, Stabilization of concentration profiles in colalyst particles, J. Differential Equations, 20 (1976), pp. 356-368.

[20] N. D. AlikAkos, Quantitotive maximum principles and strongly coupled gradient-like reaction-diffusion systems, Proc. Roy. Soc. Edinburgh, 94A (1983), pp. 265-286.

[21] F. J. MANCEBO AND \$. M. VEGA, Glabal analysis of a model of porous catalyst, 1992, in preparation.

[22] S. N. Chow AND J. K. HALE, Methods of Bifurcation Theory, Springer-Verlag, Berlin, New York, 1982.

[23] F. J. Mancrio, Efectos termicos incipientes en catalizadores porosos, Tesis Doctoral, Universidad Politêcnica de Madrid, Madrid, Spain, 1992.

[24] E. CONWAY, D. HOFF, ANIJ J. SMOLLER, Large-time behavior of solutions of systems of nonlinear reaction-diffusion equation, SIAM \&. Appl. Math., 35 (1978), pp. 1-16.

[25] Y. Kuramoto, Chemical Oscillations, Waves and Turbulence, Springer-Verlag, Berlin, New York, 1984

[26] W. F. LANGFord, Periodic and steady mode interaction lead to tori, SYAM J. Appl. Math., 37 (1979), pp. $22-48$.

[27] G. looss AND W. F. LANGFORD, Conjectures and the routes to turbulence via bifurcation, in Nonlinear Dynamics, R. H. G. Helleman, ed., New York Academy of Sciences, New York, 1980, pp. 489-505.

[28] P. FIEgUTH AND E. WICKE, Der Übergang vom Zünd/Lösch-Verhalen au stabilen Reaktionszuständen bei einem adiabatischen Rohrreaktor, Chemie-Ingr-Tech, 43 (1971), pp. 604-608.

[29] H. BEUSCH, P. FIEGUTH, AND E. WiCKE, Unstable behatior of chemical reactions af single catalyst particles, in Proc. First Intemat. Conf. on Chemical Reaction Engineering, Washington, DC, 1970 , American Chemical Society, Washington, DC, pp. 615-621.

[30] F. J. MANCE.3O AND J. M. VEGA, An asymptotic justification of a model of isothermal catalyst, J. Math. Anal. Appl., 1992, to appear.

[31] A. Di LidDO ano L. MadDalena, Mathematical analysis of a chemical reaction with iumped temperature and strong absorption, J. Math. Anal. Appl., 1991, to appear. 